\title{
Physical Models and Dimensional Traceability of WR15 Rectangular Waveguide Standards for Determining Systematic Uncertainties of Calibrated Scattering-Parameters
}

\author{
Jeffrey A. Jargon \\ Dylan F. Williams \\ Angela C. Stelson \\ Christian J. Long \\ Aaron M. Hagerstrom \\ Paul D. Hale \\ John R. Stoup \\ Eric S. Stanfield \\ Wei Ren
}


NIST Technical Note 2109

\title{
Physical Models and Dimensional Traceability of WR15 Rectangular Waveguide Standards for Determining Systematic Uncertainties of Calibrated Scattering-Parameters
}

\author{
Jeffrey A. Jargon \\ Dylan F. Williams \\ Angela C. Stelson \\ Christian J. Long \\ Aaron M. Hagerstrom \\ Paul D. Hale \\ RF Technology Division \\ John R. Stoup \\ Eric S. Stanfield \\ Wei Ren \\ Sensor Science Division \\ Physical Measurement Laboratory
}

Communications Technology Laboratory

This publication is available free of charge from:

https://doi.org/10.6028/NIST.TN.2109

August 2020

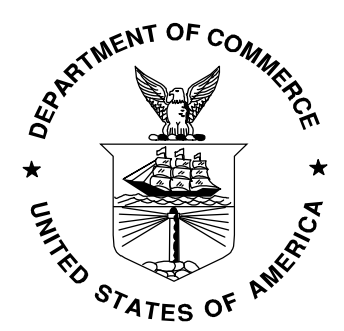

U.S. Department of Commerce Wilbur L. Ross, Jr., Secretary

National Institute of Standards and Technology Walter Copan, NIST Director and Undersecretary of Commerce for Standards and Technology 
Certain commercial entities, equipment, or materials may be identified in this document in order to describe an experimental procedure or concept adequately. Such identification is not intended to imply recommendation or endorsement by the National Institute of Standards and Technology, nor is it intended to imply that the entities, materials, or equipment are necessarily the best available for the purpose.

National Institute of Standards and Technology Technical Note 2109 Natl. Inst. Stand. Technol. Tech. Note 2109, 41 pages (August 2020) CODEN: NTNOEF

This publication is available free of charge from: https://doi.org/10.6028/NIST.TN.2109 


\begin{abstract}
In this report, we document the models and dimensional traceability of our WR 15 rectangular waveguide standards for performing multiline thru-reflect-line calibrations from $50 \mathrm{GHz}$ to $75 \mathrm{GHz}$ using vector network analyzers. We identify the equations used in our models of the transmission-line standards and present a method for determining a waveguide's metal conductivity using a closed-form solution relating it to the propagation constant. Next, we provide details of the traceable dimensional measurements and associated uncertainties of our WR15 transmission line standards. And finally, we describe how our software, the NIST Microwave Uncertainty Framework, is used to implement physical models of calibration standards and propagate these systematic uncertainties to the calibrated scattering-parameters of a device under test. We include a measurement example for illustrative purposes.
\end{abstract}

\title{
Key words
}

Calibration; dimensional traceability; measurement; models; rectangular waveguide; standards; systematic uncertainties; vector network analyzer. 
Table of Contents

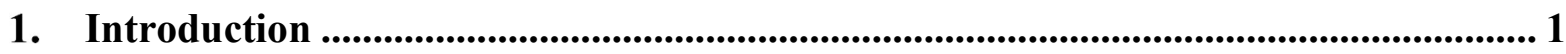

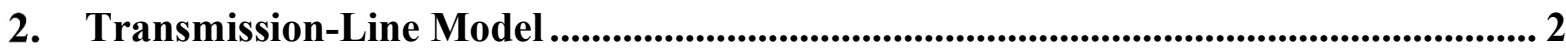

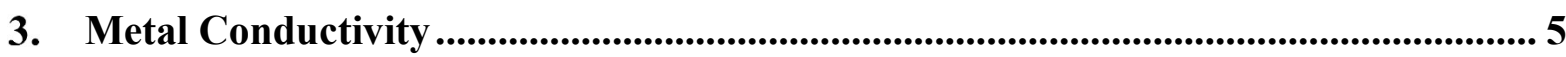

4. Junctions with Unequal Heights and Widths....................................................... 6

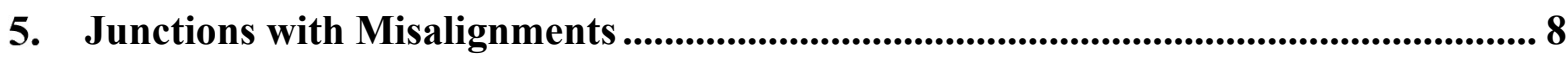

6. Dimensional Measurements..................................................................................... 10

7. Software Implementation.................................................................................... 13

References .................................................................................................................... 26

Appendix: Pseudo-Code for Calculating Lateral and Angular Displacements .............. 28 


\section{Introduction}

The mission of the National Institute of Standards and Technology (NIST) is to promote U.S. innovation and industrial competitiveness by advancing measurement science, standards, and technology in ways that enhance economic security and improve our quality of life [1]. To help meet the needs of the nation, NIST provides numerous measurement services that assist customers in establishing metrological traceability to fundamental constants. Such traceability requires an unbroken chain of measurements and uncertainties for each link in the chain [2].

One such measurement service provided by NIST is microwave scattering-parameters (Service ID 61290S) [3]. Scattering-parameters ( $S$-parameters) describe the behavior of linear networks when undergoing steady-state stimuli by electrical signals. Customers may send in one- and two-port artifacts with a variety of coaxial or rectangular waveguide connectors, and NIST provides traceable measurements at user-selected frequencies appropriate for the connector type by using a calibrated vector network analyzer (VNA).

Although numerous calibration techniques for VNAs are available, multiline thru-reflect-line (TRL) is perhaps the most fundamental and accurate method [4]. Multiline TRL utilizes an ensemble of uncorrected two-port $S$-parameter measurements with switch terms [5] or complex forward and backward wave-parameters [6] collected from a set of calibration artifacts. These measurements are used to compute correction coefficients. These coefficients can then be used to correct the measured $S$-parameters or wave-parameters of a device under test (DUT). While it is more common to simply correct $S$-parameters, the wave-parameters are more general and allow for modulated and large-signal measurements to be supported by the calibration.

Multiline TRL is based on transmission-line standards that differ only in length and an arbitrary reflect standard that is identical for both port connections. Making use of multiple transmission-line standards enables redundancy for the suppression of random errors and permits calibration over a wide frequency band. Furthermore, multiline TRL determines the propagation constant of the line standards. This can facilitate determining the frequencydependent characteristic impedance and transforming the calibration's reference impedance to a desired reference impedance, such as the impedance of an ideal guide [7].

$S$-parameters are, by definition, unitless quantities defined by ratios of the wave-parameters. The wave-parameters are determined through magnitude and phase readings, but since they are ratioed, the only requirement is that the magnitude and phase measurements are linear. The traceability path for corrected $S$-parameters depends on the calibration method. In the case of multiline TRL, $S$-parameters are traceable to dimensions of the transmission-line standards, namely each line's length and cross-sectional geometry. For coaxial standards, the crosssectional dimensions of interest are the outer conductors' inner diameters and the inner conductors' outer diameters. For rectangular waveguide standards, the heights and widths of the waveguide apertures are required.

Here, we document the models and dimensional traceability of our WR15 rectangular waveguide standards used for performing multiline TRL calibrations. The WR15 designation refers to a size that is specified to have dimensions of $3.7592 \mathrm{~mm}$ ( 0.148 inches) by 1.8796 $\mathrm{mm}$ (0.074 inches), a cutoff frequency of approximately $39.9 \mathrm{GHz}$, and a normal operating 
range from $50 \mathrm{GHz}$ to $75 \mathrm{GHz}$. In Section 2, we provide the equations used in our model of the transmission-line standards. These models are used for determining systematic uncertainties of each standard and serve as the basis for propagating them through the calibration algorithm to the calibrated $S$-parameters of a DUT. In Section 3, we present a method for determining the waveguide's metal conductivity using a closed-form solution relating it to the propagation constant. In Section 4, we review the equations used to account for height and width differences between two waveguides when they are joined, such as when a transmission line is connected to a test port of a VNA. In Section 5, we review the equations used to account for lateral and angular misalignments between two waveguides when they are connected. In Section 6, we provide details of the traceable dimensional measurements and associated uncertainties of our WR15 transmission line standards. And in Section 7, we describe how our software, the NIST Microwave Uncertainty Framework [8], is used to implement physical models of calibration standards and automatically propagate the uncertainties to the calibrated $S$-parameters of a DUT. We include a measurement example for illustrative purposes.

\section{Transmission-Line Model}

A rectangular waveguide is a hollow metallic structure of length $l$ with rectangular cross section. The conducting walls confine electromagnetic waves to the interior of the waveguide. The frequencies of the electromagnetic waves that can be propagated are determined by the guide dimensions, specifically width $a$ and height $b$, as shown in Fig. 1. The width $a$ is usually, but not always, set to twice the height $b$ to maximize the bandwidth of the waveguide. Rectangular waveguides offer advantages over other transmission media, such as coaxial, microstrip, and coplanar waveguide, in that they are completely shielded, can transmit high powers, and have very low loss due to the air dielectric. Disadvantages of waveguide include high cost, the inability to pass direct currents (DC) along with radiofrequency (RF) signals, and a more limited fractional bandwidth of single-mode propagation than many other transmission media.

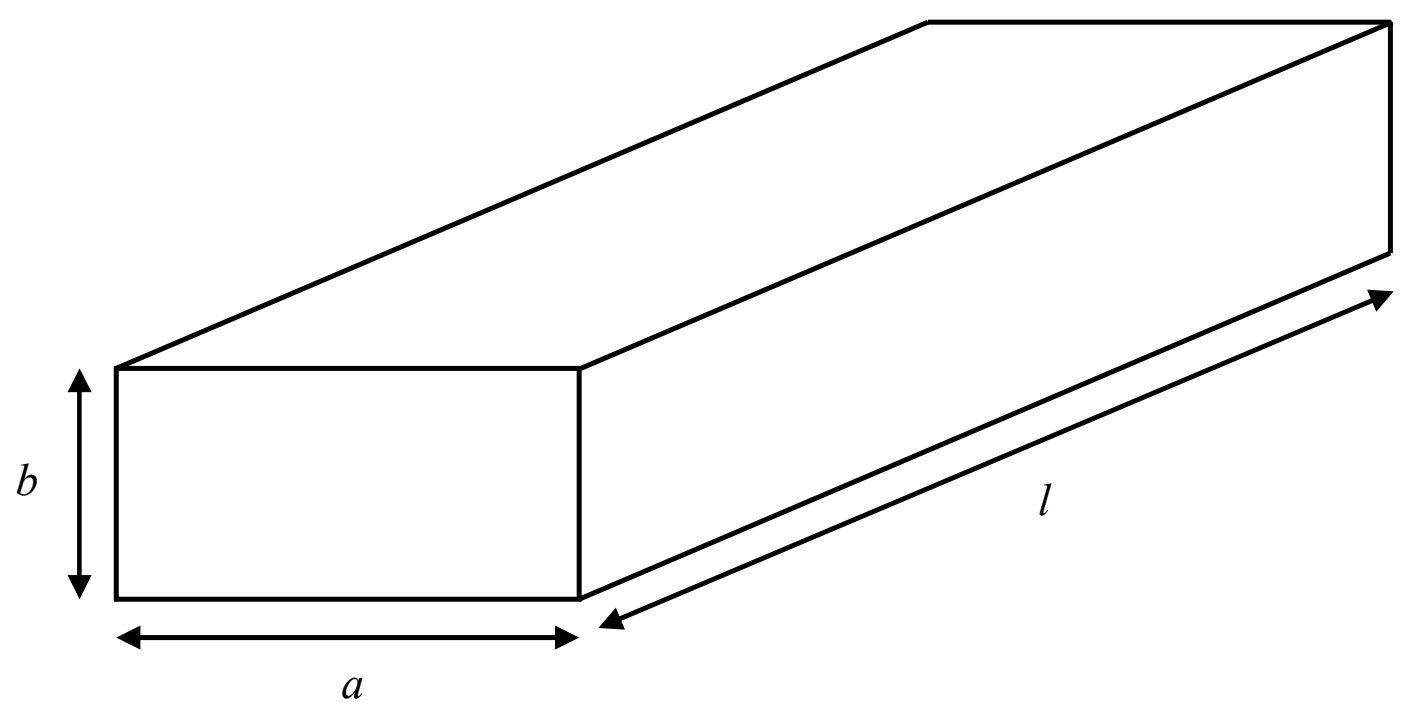

Fig. 1. Dimensions of a rectangular waveguide. 
Because waveguides use a single conductor, transverse electromagnetic (TEM) modes of transmission are not possible. The types of waves that can be propagated are transverse electric (TE) and transverse magnetic (TM) modes. The modes are labeled by integer subscripts $n$ and $m$ (i.e., $\mathrm{TE}_{n m}$ ), which refer to the number of standing-wave interference maxima occurring in the fields along the two transverse coordinates. Typically, a waveguide is operated so that only the lowest-order mode is present. In the case of rectangular waveguide, the lowest order mode is $\mathrm{TE}_{10}$.

In this section, we summarize the various equations used for modeling the $S$-parameters of a rectangular waveguide section of line operating in the $\mathrm{TE}_{10}$ mode [9]. The $S$-parameters are directly a function of the propagation constant $\gamma$ and the line length $l$. The propagation constant is comprised of the phase constant $\beta_{10}$ and attenuation constant $\alpha_{10}$, which are, in turn, dependent on the wave number in free space $k_{0}$, the cutoff wave number $k_{c, 10}$, the characteristic impedance of free space $Z_{0}$, the conductivity $\sigma$ of the waveguide walls, and the dimensions $a$ and $b$. Furthermore, we account for the possibility of the waveguide having rounded inside corners rather than perfect $90^{\circ}$ corners.

The cutoff frequency $f_{c, n m}(\mathrm{~Hz})$ for the $n m$ mode represents the lowest frequency the mode can propagate in the waveguide and is dependent on its physical dimensions. It is given by

$$
f_{c, n m}=\frac{c}{2 \pi}\left[\left(\frac{n \pi}{a}\right)^{2}+\left(\frac{m \pi}{b}\right)^{2}\right]^{1 / 2},
$$

where $c=2.99792458 \times 10^{8} \mathrm{~m} / \mathrm{s}$ is the speed of light. For the $\mathrm{TE}_{10}$ mode,

$$
f_{c, 10}=\frac{c}{2 a} .
$$

The wave number in free space $k_{0}$ is a variable defined from the Helmholtz equation, which is derived from Maxwell's equations, and is given by

$$
k_{0}=\omega \sqrt{\mu_{0} \varepsilon_{0}},
$$

where $\omega=2 \pi f$ is the radial frequency, $f$ is the frequency in $\mathrm{Hz}, \mu_{0}=1.25663706212 \times 10^{-6} \mathrm{H} / \mathrm{m}$ is the permeability of free space [10], and $\varepsilon_{0}=8.8541878128 \times 10^{-12} \mathrm{~F} / \mathrm{m}$ is the permittivity of free space [11].

Whereas the wave number $k_{0}$ corresponds to a uniform plane wave, the cutoff wave number $k_{c, n m}$ for the $n m$ mode depends on the boundary conditions for a particular waveguide type. For rectangular waveguide $k_{c, n m}$ takes on certain quantized values that depend on its physical dimensions, and is given by

$$
k_{c, n m}=\left[\left(\frac{n \pi}{a}\right)^{2}+\left(\frac{m \pi}{b}\right)^{2}\right]^{1 / 2} .
$$

For the $\mathrm{TE}_{10}$ mode, 


$$
k_{c, 10}=\frac{\pi}{a}
$$

The phase constant $\beta_{n m}(1 / \mathrm{m})$ of the $n m$ mode is the imaginary component of the waveguide's propagation constant and represents the change in phase per unit length along the path travelled by the wave at any instant. It is given by

$$
\beta_{n m}=\left[k_{0}^{2}-k_{c, n m}^{2}\right]^{1 / 2}
$$

For the $\mathrm{TE}_{10}$ mode,

$$
\beta_{10}=\left[k_{0}^{2}-\left(\frac{\pi}{a}\right)^{2}\right]^{1 / 2}
$$

The characteristic impedance of free space $Z_{0}(\Omega)$ is a physical constant relating the magnitudes of the electric and magnetic fields of electromagnetic radiation travelling through free space:

$$
Z_{0}=\sqrt{\frac{\mu_{0}}{\varepsilon_{0}}} \approx 376.73 \Omega
$$

The characteristic impedance of free space $Z_{0}$ should not be confused with the $\mathrm{TE}_{10}$ wave impedance $Z_{h, 10}$ of the rectangular waveguide, which is given by

$$
Z_{h, 10}=\frac{k_{0}}{\beta_{10}} Z_{0}
$$

Assuming a finite conductivity $\sigma(\mathrm{S} / \mathrm{m})$, the waveguide walls have a surface resistance $R_{m}(\Omega)$ of

$$
R_{m}=\sqrt{\frac{\omega \mu_{0}}{2 \sigma}}
$$

for the $\mathrm{TE}_{10}$ mode. The attenuation constant $\alpha_{n m}(\mathrm{~Np} / \mathrm{m})$ of the $n m$ mode is the real component of the waveguide's propagation constant and represents the attenuation of an electromagnetic wave per unit length along the path travelled by the wave at any instant. For the $\mathrm{TE}_{10}$ mode,

$$
\alpha_{10}=\frac{R_{m}}{a b \beta_{10} k_{0} Z_{0}}\left(2 b k_{c, 10}^{2}+a k_{0}^{2}\right)
$$

The propagation constant $\gamma(1 / \mathrm{m})$ is a complex quantity that includes the phase constant and attenuation constant, and is given by

$$
\gamma=\alpha_{10}+\mathrm{j} \beta_{10}
$$

for the $\mathrm{TE}_{10}$ mode. 
For a waveguide section of line having length $l(\mathrm{~m})$, the transmission coefficients $\left(S_{21}=S_{12}\right)$ represents the ratio of the complex amplitude of the wave at the end of the waveguide to the complex amplitude at the beginning of the waveguide, and is given by

$$
S_{21}=S_{12}=e^{-\gamma l}
$$

The $\mathrm{TE}_{10}$ mode propagates forever in accordance with (2.13) without reflection in a perfect section of waveguide. Thus, an ideal section of waveguide is defined to have a reflection coefficient $\left(S_{11}=S_{22}\right)$ of zero. However, when a rectangular waveguide with rounded inside corners rather than perfect $90^{\circ}$ corners is connected to an ideal section of rectangular waveguide, the reflection coefficients $S_{11}$ and $S_{22}$ can be modeled by

$$
S_{11}=S_{22}=\left(\frac{\lambda_{g}}{a}\right)^{2} \frac{R^{2}}{a b}\left(\frac{4-\pi}{8}\right)
$$

where is $\lambda_{g}=2 \pi / \beta_{10}$ is the guide wavelength $(\mathrm{m})$, and $R$ is the mean radius (m) of the rounded corners [12].

From the equations presented in this section, we see that the model of a rectangular waveguide section of line including rounded corners (Eq. (2.13) and (2.14)) requires the following variables: frequency $f$, length $l$, width $a$, height $b$, conductivity $\sigma$, and radius of the rounded corners $R$.

\section{Metal Conductivity}

From the previous section, we see that one of the inputs required for the model of a rectangular waveguide section of line is the metal conductivity $\sigma$. This value can be determined experimentally using a closed-form solution relating the propagation constant to the conductivity and taking the mean value over the measured frequencies. Assuming the conductivity for all the lines is the same for a single kit from a given manufacturer, we first perform a multiline TRL calibration [4], which calculates the frequency-dependent values for

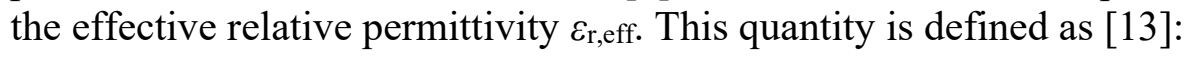

$$
\varepsilon_{\mathrm{r}, \mathrm{eff}}=-\left(\frac{c \gamma}{\omega}\right)^{2}
$$

Solving for the propagation constant $\gamma$ gives

$$
\gamma=\sqrt{-\varepsilon_{\mathrm{r}, \mathrm{eff}}}\left(\frac{\omega}{c}\right)
$$

Here, it is important to check the sign of $\gamma$ to make sure the root choice is correct. For passive devices, the real part, corresponding to the attenuation constant, should be positive so that energy is not created. The attenuation constant $\alpha_{10}$ is thus given by

$$
\alpha_{10}=\operatorname{Re}\{\gamma\}
$$


Next, by rearranging Eq. (2.11) we can solve for the surface resistance $R_{m}$ :

$$
R_{m}=\frac{\alpha_{10}}{2 b k_{c, 10}^{2}+a k_{0}^{2}}\left(a b \beta_{10} k_{0} Z_{0}\right) .
$$

And finally, Eq. (2.10) can be rearranged to solve for the metal conductivity:

$$
\sigma=\frac{\omega \mu_{0}}{2 R_{m}^{2}} .
$$

The values of $\sigma$ calculated at each of the measured frequencies may be used to determine a mean value and a standard deviation.

\section{Junctions with Unequal Heights and Widths}

When two rectangular waveguide devices are joined, differences in height or width will result in an impedance mismatch. In this section, we summarize the equations for two cases: (1) a junction with equal widths and unequal heights, and (2) a junction with equal heights and unequal widths [14].

A junction between two rectangular waveguides with equal widths and unequal heights is illustrated in Fig. 2. The difference in the heights $b$ and $b^{\prime}$ (Fig. 2a) results in a capacitance $B$ (Fig. 2b). First, the ratio $\alpha$ of the heights is calculated as

$$
\alpha=\frac{b^{\prime}}{b} .
$$

Note that $\alpha$ in this section does not represent the attenuation constant, but we are using this symbol to remain consistent with the notation in reference [14]. Next, $\delta$ is defined as

$$
\delta=1-\alpha .
$$

For $\delta<<1$, meaning the difference between the two heights is very small, the capacitance $B$ normalized to the ideal $\mathrm{TE}_{10}$ wave admittance $Y_{h, 10}\left(1 / Z_{h, 10}\right)$ can be approximated as

$$
\frac{B}{Y_{h, 10}} \approx \frac{2 b}{\lambda_{g}}\left(\frac{\delta}{2}\right)^{2}\left[\frac{2 \ln \left(\frac{2}{\delta}\right)}{1-\delta}+1+\frac{17}{16}\left(\frac{b}{\lambda_{g}}\right)^{2}\right] .
$$

The impedance-transform of the $\mathrm{TE}_{10}$ wave impedance due to the change in height at the

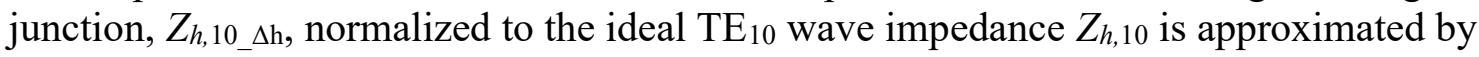

$$
\frac{Z_{h, 10 \_\Delta \mathrm{h}}}{Z_{h, 10}} \approx \alpha .
$$




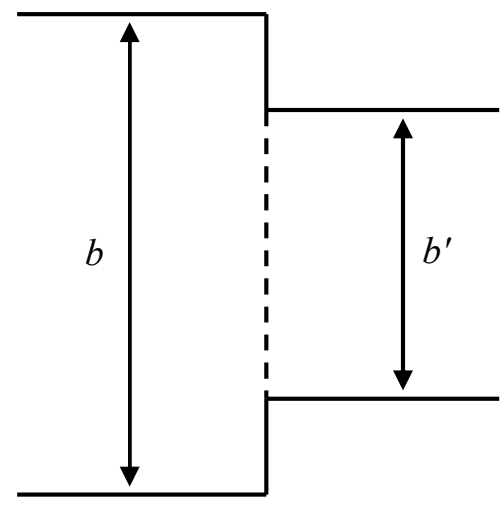

(a) Side view.

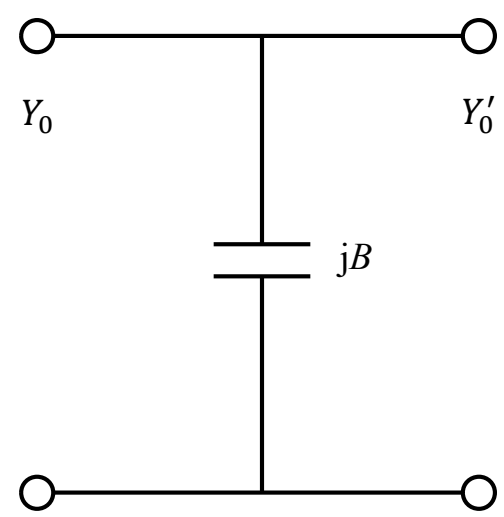

(b) Equivalent circuit.

Fig. 2. Junction between two rectangular waveguides with equal widths and unequal heights.

A junction between two rectangular waveguides with equal heights and unequal widths is illustrated in Fig. 3. The difference in the widths $a$ and $a^{\prime}$ (Fig. 3a) results in an inductance $B$ (Fig. 3b). First, the ratio $\alpha$ of the widths is calculated as

$$
\alpha=\frac{a^{\prime}}{a}
$$

Next, $\beta$ is defined as

$$
\beta=1-\alpha
$$

In a similar fashion to $\alpha$, note that $\beta$ in this section does not represent the phase constant, but we are using this symbol to remain consistent with the notation in reference [14]. For $\beta<<1$, meaning the difference between the two widths is very small, the inductance $B$ normalized to the ideal TE 10 wave admittance $Y_{h, 10}\left(1 / Z_{h, 10}\right)$ can be approximated as

$$
\frac{B}{Y_{h, 10}} \approx-\frac{\lambda_{g}}{2 a}\left[\frac{\beta^{2}(1+\beta) \ln \left(\frac{2}{\beta}\right)}{1-\frac{\beta}{2}}\right]\left[1-\frac{27}{8} \frac{Q+Q^{\prime}}{1+8 \ln \left(\frac{2}{\beta}\right)}\right]
$$

where

$$
Q=1-\sqrt{1-\left(\frac{2 a}{3 \lambda_{g}}\right)^{2}}
$$

and 


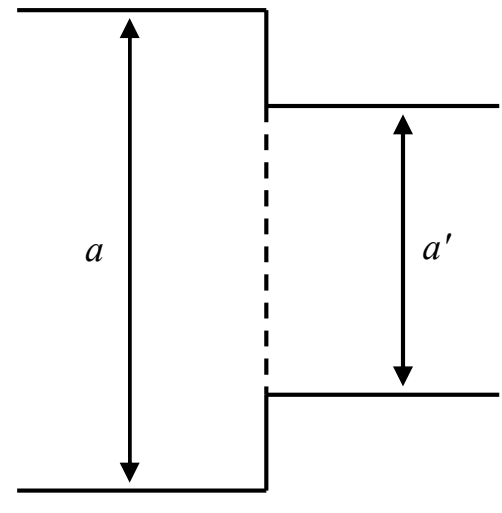

(a) Top view.

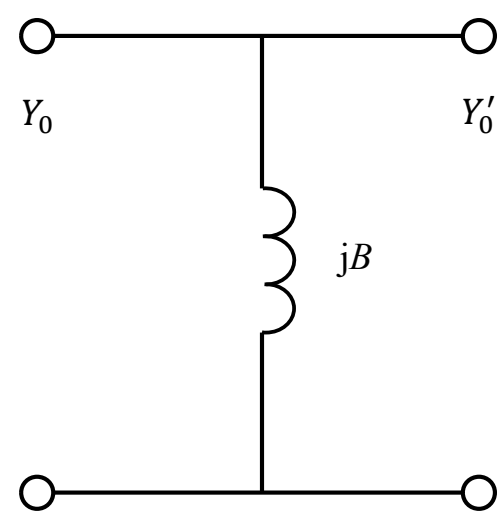

(b) Equivalent circuit.

Fig. 3. Junction between two rectangular waveguides with equal heights and unequal widths.

$$
Q^{\prime}=1-\sqrt{1-\left(\frac{2 a^{\prime}}{3 \lambda_{g}}\right)^{2}}
$$

The impedance-transform of the $\mathrm{TE}_{10}$ wave impedance due to the change in width at the junction $Z_{h, 10 \_\Delta \mathrm{w}}$ normalized to the ideal $\mathrm{TE}_{10}$ wave impedance $Z_{h, 10}$ is approximated by

$$
\frac{Z_{h, 10 \_\Delta \mathrm{w}}}{Z_{h, 10}} \approx \frac{\lambda_{g}^{\prime} a^{\prime}}{\lambda_{g} a}\left(1+\beta+\frac{\beta^{2}}{2}\right)
$$

\section{Junctions with Misalignments}

When two rectangular waveguide devices are joined, any misalignment will result in an impedance mismatch [15]. Specifically, E-plane (height), $H$-plane (width), and angular displacements may cause systematic offsets. At high frequencies, these misalignments can result in significant uncertainties [16].

In reference [15], Hunter used modal analysis to calculate the reflections for offsets in the $E$ plane and $H$-plane. He provided approximate formulas for displacements of up to $25 \%$ of the waveguide dimensions.

For an $E$-plane displacement of distance $s$, the magnitude of the reflection coefficient $\Gamma$ can be approximated as

$$
\log _{10}(|\Gamma|) \approx \sum_{n=0}^{3} u_{n}(\xi-\alpha)^{n} \log _{10}(\tau)+\sum_{n=0}^{3} v_{n}(\xi-\alpha)^{n}
$$


where $\xi=b / \lambda_{g}, \alpha=0.3, \tau=s / b$, and the coefficients $u_{n}$ and $v_{n}$ are given in columns two and three of Table 1. The normalized admittance $\left(Y_{0}=Z_{0}{ }^{-1}\right)$ for the $E$-plane displacement can be calculated as

$$
\left(\frac{B}{Y_{0}}\right)_{E}=\frac{2 \Gamma}{\sqrt{1-\Gamma^{2}}}
$$

For an $H$-plane displacement of distance $s$, the magnitude of the reflection coefficient $\Gamma$ can be approximated with Eq. (5.1), where $\xi=a / \lambda_{g}, \alpha=0.7, \tau=s / a$, and the coefficients $u_{n}$ and $v_{n}$ are given in columns four and five of Table 1 . The normalized admittance for the $H$-plane displacement $\left(B / Y_{0}\right)_{H}$ can be calculated with Eq. (5.2).

In reference [16], Williams used a commercial finite-element-method solver to calculate the impedance mismatch for an angular offset $\varphi$ at the interface between two rectangular waveguides. He provided the following approximate formula to determine the normalized admittance $\left(B / Y_{0}\right)_{\varphi}$ :

$$
\left(\frac{B}{Y_{0}}\right)_{\varphi} \approx-\left[0.000225 \varphi^{2}+\left(0.01+0.0049 \varphi^{2}\right)\left|\frac{a}{\lambda_{0}}-0.9\right|^{2}\right]
$$

where $\lambda_{0}=c / f$ is the free-space wavelength.

The overall normalized admittance $\left(B / Y_{0}\right)_{M}$ due to misalignments is simple the sum of the three contributions:

$$
\left(\frac{B}{Y_{0}}\right)_{M}=\left(\frac{B}{Y_{0}}\right)_{E}+\left(\frac{B}{Y_{0}}\right)_{H}+\left(\frac{B}{Y_{0}}\right)_{\varphi}
$$

Table 1. Hunter's polynomial coefficients for approximation to $|\Gamma|$ In Eq. (5.1).

\begin{tabular}{|c|c|c|c|c|}
\hline \multirow{2}{*}{$N$} & \multicolumn{2}{|c|}{$E$-Plane Displacement } & \multicolumn{2}{c|}{$H$-Plane Displacement } \\
\cline { 2 - 5 } & $u_{n}$ & $v_{n}$ & $u_{n}$ & $v_{n}$ \\
\hline 0 & 1.833 & 0.293 & 1.75 & 0.635 \\
1 & 0.276 & 2.133 & -0.332 & -1.562 \\
2 & 0.73 & 0.78 & -2.71 & 0.44 \\
3 & 0 & 19.69 & -3.57 & -7.63 \\
\hline
\end{tabular}




\section{Dimensional Measurements}

Our WR15 calibration kit, manufactured by Flann Microwave ${ }^{1}$, consists of nine brass shims with lengths ranging from $1.610 \mathrm{~mm}$ to $7.789 \mathrm{~mm}$, which are used as airlines for multiline TRL calibrations, as well as two commercially available test ports consisting of straight sections of waveguide. The top view of the circular-shaped shims, shown in Fig. 4, are known as two-pin flanges since the two guide-pin holes, labeled ' $G$ ', are used for alignment with other devices using guide pins. The shims and test ports were measured by the NIST Dimensional Metrology Group in Gaithersburg, MD [17] using a coordinate measuring machine (CMM) error-mapped with a traceable, stabilized $\mathrm{HeNe}$ laser system. The $\mathrm{CMM}$ was housed in a constant-humidity environment where the temperature was controlled to $20.00 \pm 0.03^{\circ} \mathrm{C}$.

The lengths of the shims were measured using an invar micrometer combined with a calibrated $\mathrm{HeNe}$ laser displacement system. The measurements were made near the center slot areas of the shims. The standard uncertainty for each shim length measurement was specified as 0.5 $\mu \mathrm{m}$. Table 2 lists the measured values of length along with uncertainty for the nine shims, as designated by serial number.

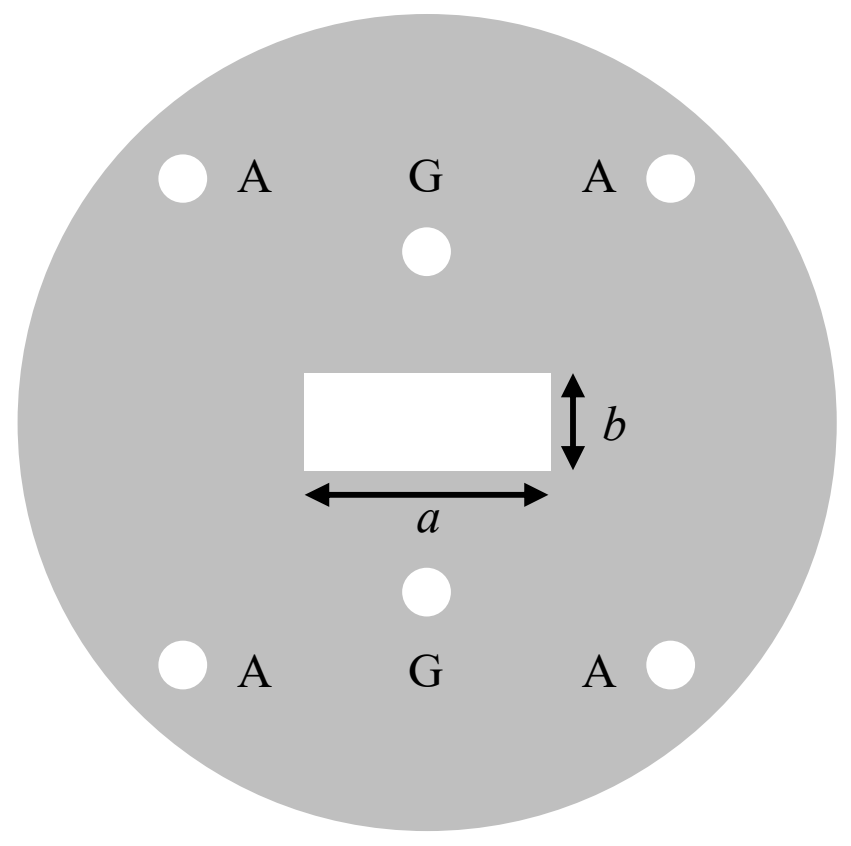

Fig. 4. Top view of a WR15 waveguide shim. The center slot has width $a$ and height $b$. The four holes labeled ' $A$ ' are outer alignment holes and the two holes labeled ' $G$ ' are guide pin holes.

\footnotetext{
${ }^{1}$ The National Institute of Standards and Technology does not endorse commercial products. Other products may work as well or better.
} 
Table 2. Length $(l)$ measurements of shims.

\begin{tabular}{|c|c|}
\hline $\begin{array}{c}\text { Serial } \\
\text { Number }\end{array}$ & $\begin{array}{c}\text { Length } \pm \text { Std. Unc. } \\
(\mathrm{mm})\end{array}$ \\
\hline 00620 & $1.610 \pm 0.0005$ \\
100013 & $1.612 \pm 0.0005$ \\
210330 & $7.789 \pm 0.0005$ \\
210332 & $4.673 \pm 0.0005$ \\
210333 & $4.673 \pm 0.0005$ \\
210334 & $3.113 \pm 0.0005$ \\
210335 & $3.114 \pm 0.0005$ \\
210336 & $1.557 \pm 0.0005$ \\
210337 & $1.553 \pm 0.0005$ \\
\hline
\end{tabular}

The widths $a$ and heights $b$ of each device were measured at the front and back planes. The standard uncertainty for these measurements was specified to be $0.13 \mu \mathrm{m}$, except for two shims (serial numbers 210334 and 210337). These two exceptions indicated more inconsistency than was evident in the others, possibly due to contamination or interior surface damage, and were specified to have a standard uncertainty of $0.26 \mu \mathrm{m}$. Table 3 lists the measured values of the shims' and test ports' widths at the front and back planes along with uncertainties, and Table 4 lists the values of the heights.

Rather than tracking each of the devices' individual measurements, we used the specified values of $3.7592 \mathrm{~mm}$ for the width and $1.8796 \mathrm{~mm}$ for the height, and determined the uncertainties by calculating the root-mean-squares (RMS) of the widths and heights from the measured values listed in Tables 3 and 4 . This resulted in standard uncertainties of \pm 0.0035 $\mathrm{mm}$ for the widths and $\pm 0.0029 \mathrm{~mm}$ for the heights.

We chose to use the specified values of width and height for all the shims as opposed to the individually measured values because the multiline TRL algorithm is not compatible with individually measured widths and heights. The multiline TRL algorithm is considered the most accurate calibration algorithm because the self-defined models it employs do not rely on approximate analytic expressions for the $S$-parameters of the shims, but rather only on measured $S$-parameters and lengths of the shims. This is a subtle point - multiline TRL does not use any other parameters or error mechanisms to determine the nominal calibration, rather they are only used for determining uncertainties.

The internal corner radii for each shim were measured using a commercial vision instrument with variable magnification. Both ends of the shims were imaged and the results for each corner were averaged, as listed in Table 5. Measured values shown as $0.020 \mathrm{~mm}$ imply the radius was too small to reliably measure, so the number serves as a worst-case value. The standard uncertainty for the radius measurements was specified as $5 \mu \mathrm{m}$. Since the model allows only a single radius $R$, we determined a consensus value and standard uncertainty for the rounded corners of each shim. To accomplish this, we employed the NIST Consensus Builder [18, 19], a web-based program developed for combining measurement results and calculating a 
consensus estimate along with an evaluation of measurement uncertainty that captures the stated uncertainties associated with the individually measured values in addition to a component that accounts for the combination of multiple measurement results. Three methods of data reduction are available in the software. We used the Linear Pool method [19] since it "has been in use the longest and makes the fewest assumptions about the nature of the data." The consensus values and corresponding uncertainties for each shim radius are listed in the last column of Table 5 .

To account for junctions having $E$-plane (height), $H$-plane (width), and angular displacements between the two test ports and the shims, as discussed in Section 5, numerous dimensions were measured, including lateral displacements of the apertures, displacements of the flange holes (labeled ' $G$ ' in Fig. 4), and diameters of the flange holes. The artifact coordinate system was oriented at the top plane surface of each shim using the centerline between the flange holes defining the $+X$ direction and the symmetrical center of the flange holes defining the origin at $\mathrm{X}=0, \mathrm{Y}=0$. Rather than tracking each of the shim's individual measurements, we pooled the data and determined worst-case bounds. The aperture lateral displacements were approximately $0 \pm 0.03 \mathrm{~mm}$, the displacements of the flange holes were approximately $0 \pm$ $0.008 \mathrm{~mm}$, and the diameters of the flange holes were approximately $1.59 \pm 0.013 \mathrm{~mm}$.

The diameters of several sample guide pins were measured using a contact micrometer combined with a displacement interferometer and measured at several locations across two perpendicular planes. The average pin diameter was $1.566 \mathrm{~mm}$. The variation along any single pin was approximately $1 \mu \mathrm{m}$. The variation in average diameter among the pins was measured to be about $3.5 \mu \mathrm{m}$ with a standard deviation of about $1.5 \mu \mathrm{m}$. For the purpose of entering a pin diameter and an associated uncertainty into our models, we chose $1.566 \pm 0.005 \mathrm{~mm}$.

Table 3. Width (a) measurements of shims and test ports.

\begin{tabular}{|c|c|c|}
\hline \multirow{2}{*}{$\begin{array}{c}\text { Serial } \\
\text { Number }\end{array}$} & \multicolumn{2}{|c|}{ Width \pm Std. Unc. $(\mathrm{mm})$} \\
\cline { 2 - 3 } & Front Plane & Back Plane \\
\hline 00620 & $3.75928 \pm 0.00013$ & $3.75921 \pm 0.00013$ \\
100013 & $3.75950 \pm 0.00013$ & $3.76340 \pm 0.00013$ \\
210330 & $3.75393 \pm 0.00013$ & $3.75677 \pm 0.00013$ \\
210332 & $3.75639 \pm 0.00013$ & $3.75182 \pm 0.00013$ \\
210333 & $3.74985 \pm 0.00013$ & $3.75664 \pm 0.00013$ \\
210334 & $3.76090 \pm 0.00026$ & $3.76053 \pm 0.00026$ \\
210335 & $3.76153 \pm 0.00013$ & $3.76506 \pm 0.00013$ \\
210336 & $3.75890 \pm 0.00013$ & $3.75987 \pm 0.00013$ \\
210337 & $3.76181 \pm 0.00026$ & $3.76152 \pm 0.00026$ \\
70066 & $3.75961 \pm 0.00013$ & $3.75840 \pm 0.00013$ \\
70067 & $3.75864 \pm 0.00013$ & $3.75745 \pm 0.00013$ \\
\hline
\end{tabular}


Table 4. Height $(b)$ measurements of shims and test ports.

\begin{tabular}{|c|c|c|}
\hline \multirow{2}{*}{$\begin{array}{c}\text { Serial } \\
\text { Number }\end{array}$} & \multicolumn{2}{|c|}{ Height \pm Std. Unc. $(\mathrm{mm})$} \\
\cline { 2 - 3 } & Front Plane & Back Plane \\
\hline 00620 & $1.88042 \pm 0.00013$ & $1.88041 \pm 0.00013$ \\
100013 & $1.88091 \pm 0.00013$ & $1.88754 \pm 0.00013$ \\
210330 & $1.87763 \pm 0.00013$ & $1.88342 \pm 0.00013$ \\
210332 & $1.88340 \pm 0.00013$ & $1.87835 \pm 0.00013$ \\
210333 & $1.87554 \pm 0.00013$ & $1.88278 \pm 0.00013$ \\
210334 & $1.88088 \pm 0.00026$ & $1.88343 \pm 0.00026$ \\
210335 & $1.88467 \pm 0.00013$ & $1.88175 \pm 0.00013$ \\
210336 & $1.88077 \pm 0.00013$ & $1.88022 \pm 0.00013$ \\
210337 & $1.88012 \pm 0.00026$ & $1.88030 \pm 0.00026$ \\
70066 & $1.88000 \pm 0.00013$ & $1.87831 \pm 0.00013$ \\
70067 & $1.87897 \pm 0.00013$ & $1.87801 \pm 0.00013$ \\
\hline
\end{tabular}

Table 5. Radius $(R)$ measurements of shims.

\begin{tabular}{|c|c|c|c|c|c|}
\hline & \multicolumn{5}{|c|}{ Radius (mm) } \\
\cline { 2 - 6 } $\begin{array}{c}\text { Serial } \\
\text { Number }\end{array}$ & Corner 1 & Corner 2 & Corner 3 & Corner 4 & $\begin{array}{c}\text { Consensus Value } \\
\pm \text { Std. Dev }\end{array}$ \\
\hline 00620 & 0.029 & 0.029 & 0.030 & 0.031 & $0.030 \pm 0.005$ \\
100013 & 0.032 & 0.038 & 0.054 & 0.044 & $0.042 \pm 0.010$ \\
210330 & 0.170 & 0.190 & 0.162 & 0.163 & $0.171 \pm 0.012$ \\
210332 & 0.164 & 0.160 & 0.171 & 0.174 & $0.165 \pm 0.006$ \\
210333 & 0.183 & 0.180 & 0.189 & 0.159 & $0.178 \pm 0.012$ \\
210334 & 0.020 & 0.020 & 0.020 & 0.020 & $0.020 \pm 0.005$ \\
210335 & 0.020 & 0.020 & 0.020 & 0.020 & $0.020 \pm 0.005$ \\
210336 & 0.020 & 0.020 & 0.020 & 0.020 & $0.020 \pm 0.005$ \\
210337 & 0.020 & 0.020 & 0.020 & 0.020 & $0.020 \pm 0.005$ \\
\hline
\end{tabular}

\section{Software Implementation}

We utilized the NIST Microwave Uncertainty Framework (MUF) [8, 20-22] to develop physical models of our multiline-TRL, WR15 calibration kit. The MUF provides parallel sensitivity and Monte-Carlo analyses, and enables us to capture and propagate the significant $S$-parameter measurement uncertainties and statistical correlations between them [22]. By identifying and modeling the physical error mechanisms in the calibration standards, as we did in the previous sections, we can determine the systematic uncertainties in $S$-parameters, including their cross-frequency correlations. These uncertainties can then be propagated to measurements of the DUTs.

Figure 5 illustrates an example of how model-building is accomplished in the MUF. By bringing up the 'Model' program, the user is offered numerous choices. The top-most pulldown menu is labeled 'Model Category,' which includes 'Rectangular waveguide.' With this option chosen, the next pull-down menu, entitled 'Selection,' offers a variety of waveguide 
models including 'Section of line with rounded corners.' This model implements the equations we presented in Section 2. Immediately below the 'Selection' menu is a field called 'Name,' where the user may enter a descriptive title. And following that is a list of input mechanisms required for the chosen model. In the case we're illustrating, we need to enter parameters files for the line's width $a$, height $b$, length $l$, corner radius $R$, and metal resistivity relative to copper.

The mechanism values shown in Fig. 5 correspond to a $4.673 \mathrm{~mm}$ shim ( $\mathrm{S} / \mathrm{N} 210333)$. For each mechanism, a 'Parameter' file is required that contains a descriptive title, a mean value of the parameter and the probability-distribution limits of the parameter, as illustrated in Fig. 6. Once all the 'Parameter' files are created for a given standard, they can be dragged and dropped into the appropriate parameters in the 'Model' program, where the directory information and mean values are displayed. This model can then be saved and used as the standard definition or cascaded with additional models.

Since the dimensions of our devices were measured at a temperature of $20{ }^{\circ} \mathrm{C}$ and the temperature in our $S$-parameter laboratory is specified to be $23 \pm 2^{\circ} \mathrm{C}$, we took thermal expansion into account in determining the lengths of our brass shims. Brass has a linear thermal expansion coefficient $\left(\alpha_{L}\right)$ of approximately $19 \times 10^{-6}\left({ }^{\circ} \mathrm{C}\right)^{-1}$ [23]. We modified the length of each shim as

$$
l_{\Delta T}=l\left(1+\alpha_{L} \Delta T\right)
$$

where $l$ is the length of the shim measured at $20^{\circ} \mathrm{C}, \Delta T$ is the difference in temperature (in our case $3 \pm 2{ }^{\circ} \mathrm{C}$ ), and $l_{\Delta T}$ is the resulting length of the shim in our laboratory. Equation (7.1) can be realized in the MUF by utilizing a 'Sum of Parameters' file, as illustrated in Fig. 7. Parameters for the temperatures, measured line length, and thermal expansion coefficient are dragged and dropped into the 'List of Parameters' and the equation is entered into the area designated, 'Expression to be evaluated,' where the parameter names are contained in curly brackets. The resulting 'Sum of Parameters' file can be dragged and dropped into a 'Model' just like a 'Parameter' file.

Figures 8-10 illustrate examples of the additional models we utilized for the $4.673 \mathrm{~mm}$ shim (S/N 210333), including the two-pin waveguide flange with correlated $E$-plane/H-plane displacements, the rectangular-waveguide height change, and the rectangular-waveguide width change. The Appendix in this document includes the input quantities and pseudo-code used for calculating the lateral and angular displacements as discussed in IEEE Standard 1785.3-2016 [24] and implemented in the MUF [8] and the P1785 RWG Uncertainty Calculator [25].

Figure 11 illustrates an example of a 'Cascade' file for the $4.673 \mathrm{~mm}$ shim (S/N 210333), where the following models were cascaded: Width Change Port 1 -> Height Change Port 1 $>$ Correlated $E$ - and $H$-field Displacement Port 1 -> Transmission Line -> Correlated $E$ - and $H$-field Displacement Port 2 -> Height Change Port 2 -> Width Change Port 2.

As described in Section 3, the first step in determining the metal conductivity $\sigma$ is to perform a multiline TRL calibration, which will provide us with frequency-dependent values for the effective relative permittivity $\varepsilon_{\mathrm{r}, \text { eff. }}$ The multiline TRL calibration is set up using the "VNA 
Uncertainty Calculator' program in the MUF, as shown in Fig. 12. The pull-down menu labeled 'Main calibration' allows the user to set up the standards to be used in the calibration. The first column labeled 'Definition' is the area where the 'Model' or 'Cascade' files for the standards are dragged and dropped. The second column labeled 'Standard type' is where the standards are classified. Examples include 'Thru,' 'Switch terms,' 'Line (gamma unknown),' and 'Reflect $(\mathrm{S} 11=\mathrm{S} 22)$.' The third column labeled 'Length' is where the 'Parameter' files corresponding to the standards' lengths are placed. And the fourth column labeled 'Measurement' is where the uncorrected measurement files of the standards are placed. For two-port devices, the measurements can be either 's2p' files for $S$-parameters or 'w2p' files for wave-parameters. Note that the MUF does not provide data collection from VNAs external software is required.

In our example, we measured the wave-parameters of a flush short and several shims, five of which were used for the calibration (the serial numbers and lengths are specified in the 'Cascade' filenames shown in Fig. 12). Shim S/N 210337 was defined as the 'thru' standard. Since this standard has a length of $1.553 \mathrm{~mm}$, we were required to perform a reference-plane translation of $-0.7765 \mathrm{~mm}$, where the negative sign refers to moving the reference plane toward the VNA. This was set under the 'Options' menu. Furthermore, all the measurements taken were in the form of wave-parameters, so the switch terms did not need to be measured [6]. All measurements were taken on a frequency grid from $50 \mathrm{GHz}$ to $75 \mathrm{GHz}$ with a spacing of $50 \mathrm{MHz}$ (501 points). The VNA's IF bandwidth was set to $10 \mathrm{~Hz}$ with no averaging.

Once the calibration was performed, we obtained the frequency-dependent values $\varepsilon_{\mathrm{r}, \mathrm{eff}}-$ the real part is shown in Fig. 13 and the imaginary part is shown in Fig. 14. Since the values of the imaginary part were small relative to the noise, we fit a straight line to the data and used it for the subsequent calculations. Otherwise, we would end up with large spikes in the calculated metal conductivity. Next, we determined the attenuation constant $\alpha_{10}$ using Eqs. (3.1), (3.2), and (3.3), as shown in Fig. 15. From there, we computed $\sigma$ using Eqs. (3.4) and (3.5), as shown in Fig. 16. And finally, we calculated the mean and standard deviation of $\sigma$ over all 501 measured frequencies to be $9.0 \times 10^{6} \pm 1.0 \times 10^{6} \mathrm{~S} / \mathrm{m}$.

In the MUF, the parameter for metal resistivity is specified as relative to copper, which modifies Eq. (2.10) for the surface resistance $R_{m}(\Omega)$ as follows:

$$
R_{m}=\sqrt{\frac{\omega \mu_{0}}{2 \sigma_{\mathrm{Cu}} / L_{\mathrm{rel}}}}
$$

where $L_{\text {rel }}$ is the relative loss compared to the metal resistivity of annealed copper $\left(\sigma_{\mathrm{Cu}}=\right.$ $\left.5.8 \times 10^{7} \mathrm{~S} / \mathrm{m}\right)$. Thus, in our case, $L_{\text {rel }}=6.44 \pm 0.10$.

With this value plugged back into the models, we reran the multiline TRL calibration and corrected our DUT measurement, in this case a $4.673 \mathrm{~mm}$ shim (S/N 210332).

Figure 17 plots $\left|S_{21}\right|$ as a function of frequency along with its $95 \%$ confidence intervals, and Fig. 18 plots the corresponding individual systematic uncertainty contributions and the total 
standard uncertainty. The total standard uncertainty was less than $280 \times 10^{-6} \mathrm{~dB}$ for $\left|S_{21}\right|$. The largest contributions were the width of the longest line, the radius of the longest line, and the width of the shortest line below $63 \mathrm{GHz}$ and the $E$-plane and $H$-plane aperture displacements above $63 \mathrm{GHz}$.

Figure 19 plots the argument of $S_{21}$ as a function of frequency along with its $95 \%$ confidence intervals, and Fig. 20 plots the corresponding individual systematic uncertainty contributions and the total standard uncertainty. The total standard uncertainty was less than 0.18 degrees for $\operatorname{Arg}\left(S_{21}\right)$. The largest contributions were the widths of the longest and shortest lines below $72 \mathrm{GHz}$ and the $E$-plane and $H$-plane aperture displacements above $72 \mathrm{GHz}$.

Figure 21 plots $\left|S_{11}\right|$ as a function of frequency along with its $95 \%$ confidence intervals, and Fig. 22 plots the corresponding individual systematic uncertainty contributions and the total standard uncertainty. The largest contributions were the test port heights and widths below 72 $\mathrm{GHz}$ and the $H$-plane aperture displacements above $72 \mathrm{GHz}$.

Figures 17, 19, and 21 also compare the measured and modeled values of $\left|S_{21}\right|, \operatorname{Arg}\left(S_{21}\right)$, and $\left|S_{11}\right|$, respectively, and show good agreement. Preliminary studies indicate the overall random uncertainty is larger than the systematic components discussed in this document. 


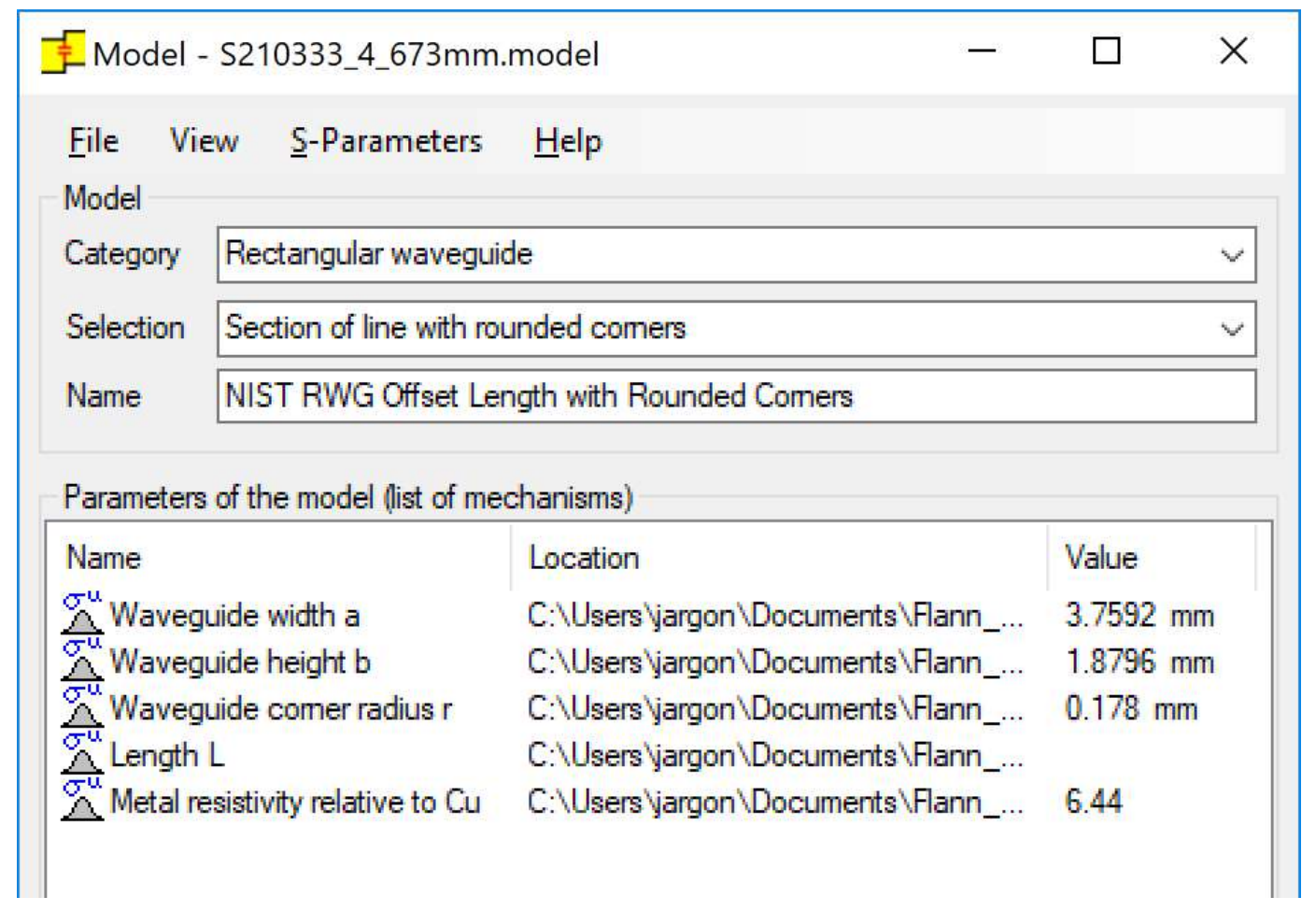

Fig. 5. MUF 'Model' file of waveguide transmission-line standard (S/N 210333) with rounded corners. Note that the 'Length' parameter does not show a corresponding value since it is represented by a 'Sum of Parameters' file.

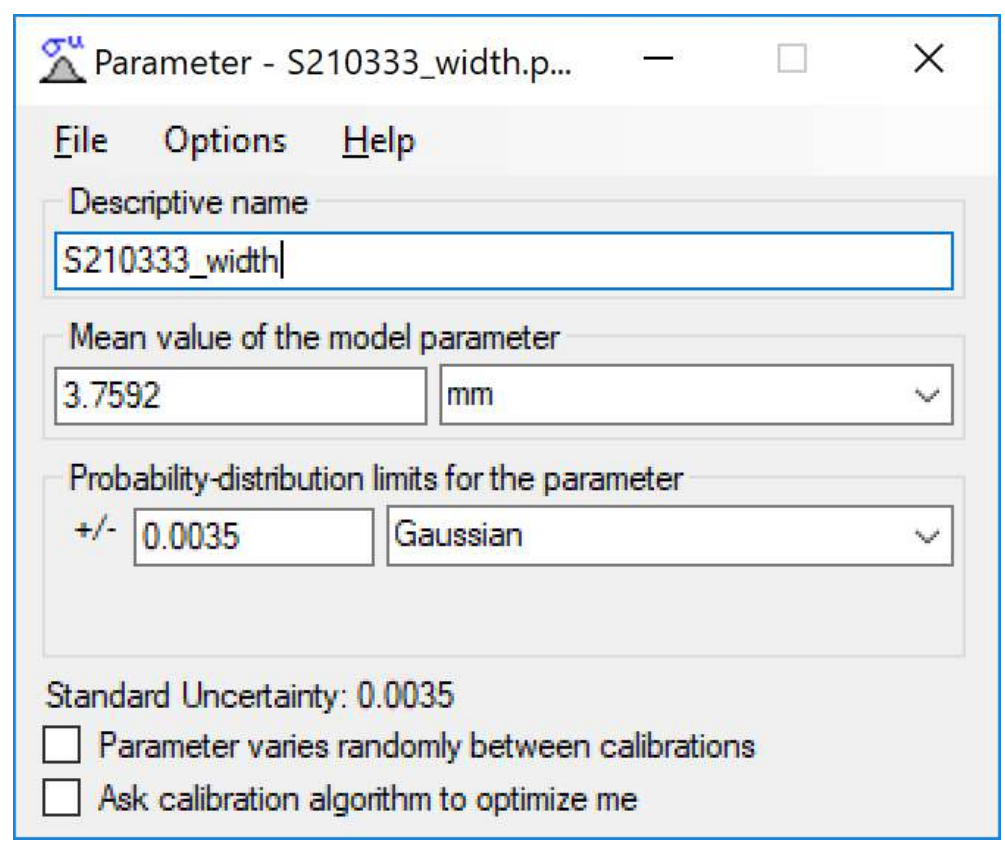

Fig. 6. MUF 'Parameter' file of transmission-line standard's width (S/N 210333). 


\begin{tabular}{|c|c|c|c|c|c|}
\hline \multicolumn{3}{|c|}{$\stackrel{\sigma^{u}}{\triangle}$ Sum of Parameters - S210333_length_temp.sumofparameters } & - & $\square$ & $x$ \\
\hline \multicolumn{5}{|l|}{ List of parameters } & \\
\hline \multirow{5}{*}{ 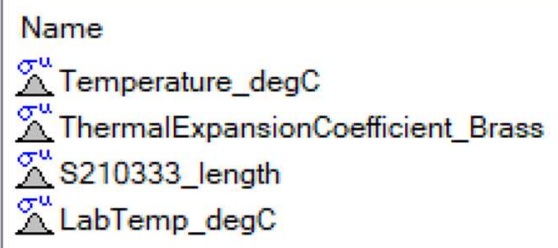 } & Location & Mean value & \multicolumn{3}{|c|}{ Dist. Limits } \\
\hline & C:IUsersljargo... & 23 & \multicolumn{3}{|c|}{$+/-2$} \\
\hline & C:IUsersljargo... & 0.000019 & \multicolumn{3}{|l|}{$+1-0$} \\
\hline & C:IUsersljargo... & $4.673 \mathrm{~mm}$ & \multicolumn{3}{|c|}{$+/-0.0005 \mathrm{~mm}$} \\
\hline & C:IUsersljargo... & 20 & \multicolumn{3}{|l|}{$+/-0$} \\
\hline \multicolumn{5}{|l|}{$<$} & $>$ \\
\hline \multicolumn{6}{|c|}{ Expression to be evaluated (Default: Sum all parameters) } \\
\hline \multicolumn{5}{|c|}{$\begin{array}{l}\{\text { S210333_length }\}^{*}\left(1+\{\text { ThermalExpansionCoefficient_Brass }\}^{*}(\{\text { Temperature_degC }\}-\right. \\
\{\text { LabTemp_degC }\}))\end{array}$} & A \\
\hline \multicolumn{5}{|c|}{ Ready to run in default directory. } & $\therefore$ \\
\hline
\end{tabular}

Fig. 7. MUF 'Sum of Parameters' file of transmission-line standard's length (S/N 210333).

\begin{tabular}{|c|c|c|c|c|}
\hline \multicolumn{5}{|c|}{ 全 Model - S210333_Flange1.model } \\
\hline File Vie & ew S-Parameters Help & & & \\
\hline Model & & & & \\
\hline Category & \multicolumn{3}{|l|}{ Rectangular waveguide } & $\checkmark$ \\
\hline Selection & \multicolumn{3}{|c|}{ Two-pin flange with correlated E-plane/H-plane displacement } & $\checkmark$ \\
\hline Name & \multicolumn{4}{|c|}{ NIST Two-Pin RWG Correlated Lateral and Angular Displacement } \\
\hline \multicolumn{5}{|c|}{ Parameters of the model (iist of mechanisms) } \\
\hline & Location & Value & $\wedge$ \\
\hline \multicolumn{2}{|c|}{$\sigma_{\sigma u}^{u}$ Waveguide width a } & C:Nusers... & $3.7592 \mathrm{~mm}$ & \\
\hline \multicolumn{2}{|c|}{$\mathrm{F}_{\mathrm{u}}^{\mathrm{u}}$ Waveguide height $\mathrm{b}$} & c: Users... & $1.8796 \mathrm{~mm}$ & \\
\hline \multicolumn{2}{|c|}{ 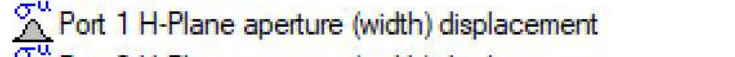 } & C: Users... & $0 \mathrm{~mm}$ & \\
\hline \multicolumn{2}{|c|}{$\mathscr{S}_{\Omega}^{u}$ Port $2 \mathrm{H}$-Plane aperture (width) displacement } & C:NUsers... & $0 \mathrm{~mm}$ & \\
\hline \multicolumn{2}{|c|}{$\stackrel{\mathrm{Su}}{\mathrm{u}}_{\mathrm{u}}^{\mathrm{P}}$ Port $1 \mathrm{E}$-Plane aperture (height) displacement } & C: Users... & $0 \mathrm{~mm}$ & \\
\hline \multicolumn{2}{|c|}{$\stackrel{\mathrm{Su}}{\mathrm{Su}}$ Port $2 \mathrm{E}$-Plane aperture (height) displacement } & C: UUsers... & $0 \mathrm{~mm}$ & \\
\hline \multirow{2}{*}{\multicolumn{2}{|c|}{ 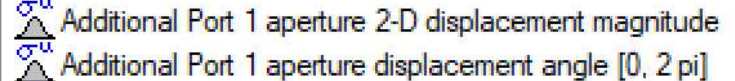 }} & C: Users... & $0 \mathrm{~mm}$ & \\
\hline & & C:U Users... & 3.14159 & \\
\hline \multicolumn{2}{|c|}{$\mathrm{gu}^{\mathrm{u}}$ Additional Port 2 aperture 2-D displacement magnitude } & C: UUsers... & $0 \mathrm{~mm}$ & \\
\hline \multirow{2}{*}{\multicolumn{2}{|c|}{$\begin{array}{l}\mathscr{S}^{\mathrm{u}} \text { Additional Port } 2 \text { aperture displacement angle }[0,2 \mathrm{pi}] \\
\text { Plange } 1 \text { hole } 12 \text {-D displacement magnitude }\end{array}$}} & C: Users... & 3.14159 & \\
\hline & & C. Users... & $0 \mathrm{~mm}$ & \\
\hline \multicolumn{2}{|c|}{$\stackrel{\square}{\triangle}$ Flange 1 hole 1 disolacement anale $[0.2$ bil } & C. Users... & 3.14159 & $\checkmark$ \\
\hline
\end{tabular}

Fig. 8. MUF 'Model' file of two-pin rectangular-waveguide flange with correlated $E$-plane $/ H$ plane displacements for transmission-line standard (S/N 210333). 


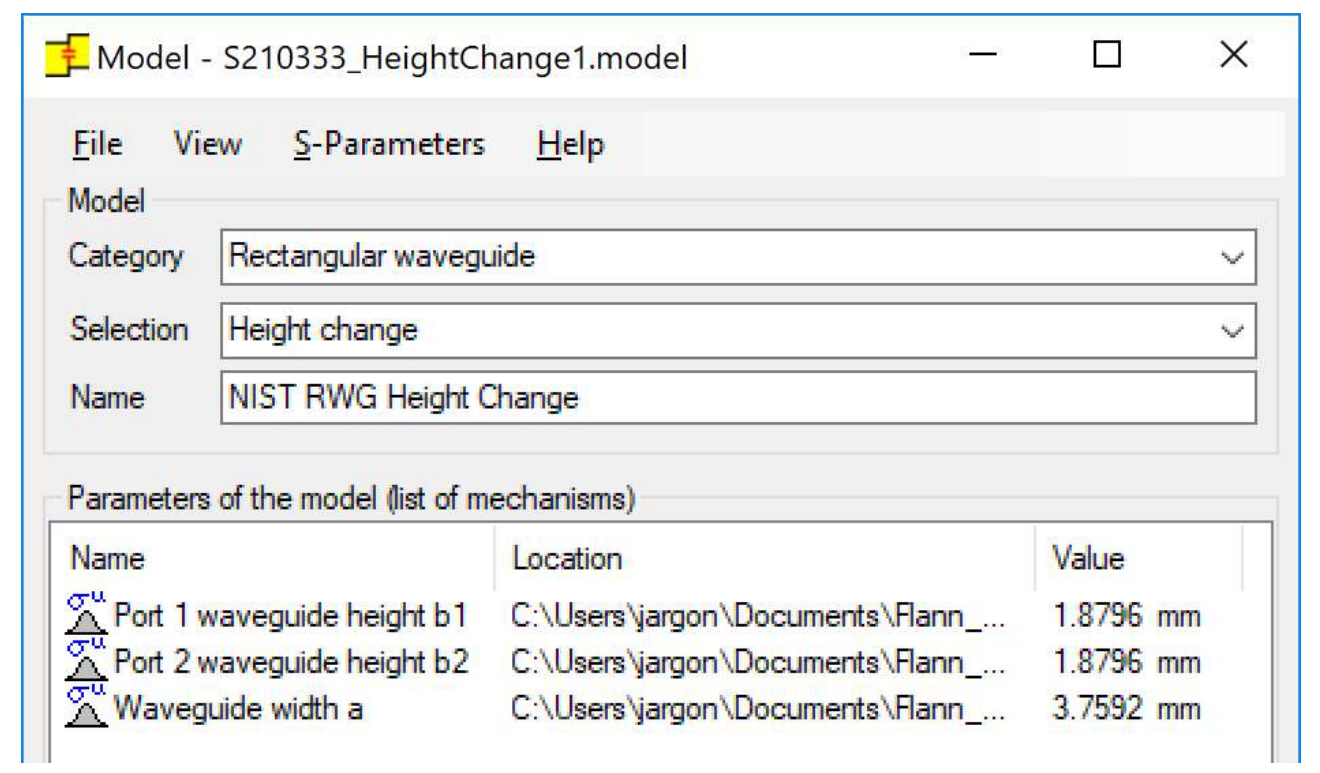

Fig. 9. MUF 'Model' file of rectangular-waveguide height change for transmission-line standard (S/N 210333).

\begin{tabular}{|c|c|c|c|c|}
\hline \multicolumn{3}{|c|}{$\frac{-}{J}$ Model - S210333_WidthChange1.model } & \multirow[t]{2}{*}{$\square$} & \multirow[t]{2}{*}{$x$} \\
\hline $\begin{array}{l}\text { File Vie } \\
\text { Model }\end{array}$ & $\underline{\text { S-Parameters }}$ & 브elp & & \\
\hline Category & \multicolumn{3}{|c|}{ Rectangular waveguide } & $\checkmark$ \\
\hline Selection & \multicolumn{3}{|l|}{ Width change } & $\checkmark$ \\
\hline Name & \multicolumn{4}{|c|}{ NIST RWG Width Change } \\
\hline \multicolumn{5}{|c|}{ Parameters of the model (list of mechanisms) } \\
\hline \multirow{3}{*}{\multicolumn{2}{|c|}{$\begin{array}{l}\text { Name } \\
و_{\triangle \mathrm{u}}^{u} \text { Port } 1 \text { waveguide width a1 } \\
\triangle \mathrm{S} \text { Port } 2 \text { waveguide width a2 }\end{array}$}} & Location & Value & \\
\hline & & C. Users yargon \Documents \Flann... & $3.7592 \mathrm{~mm}$ & \\
\hline & & C:Users \argon \Documents \Flann... & $3.7592 \mathrm{~mm}$ & \\
\hline
\end{tabular}

Fig. 10. MUF 'Model' file of rectangular-waveguide width change for transmission-line standard (S/N 210333). 


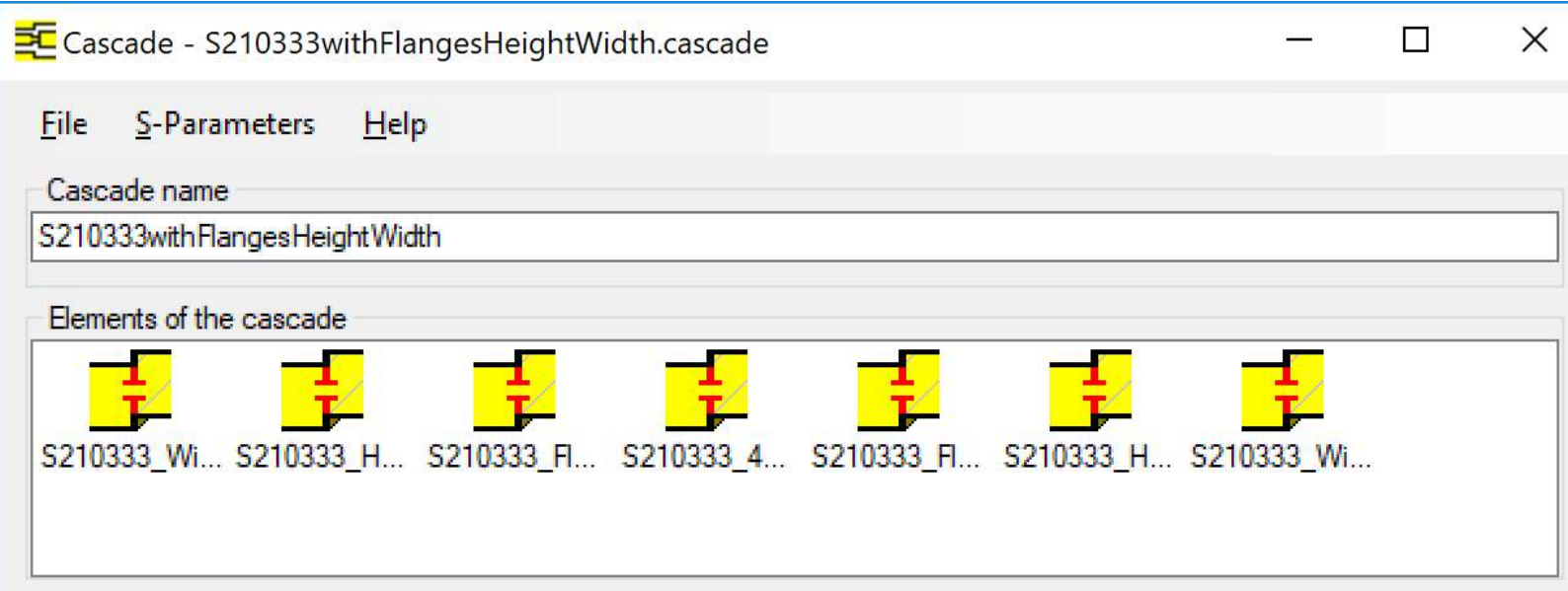

Fig. 11. MUF 'Cascade' file for rectangular-waveguide transmission-line standard (S/N 210333). The models included are Width Change Port 1 ->Height Change Port 1 -> Correlated E- and H-field Displacement Port 1 -> Transmission Line -> Correlated E- and H-field Displacement Port 2 -> Height Change Port 2 -> Width Change Port 2.

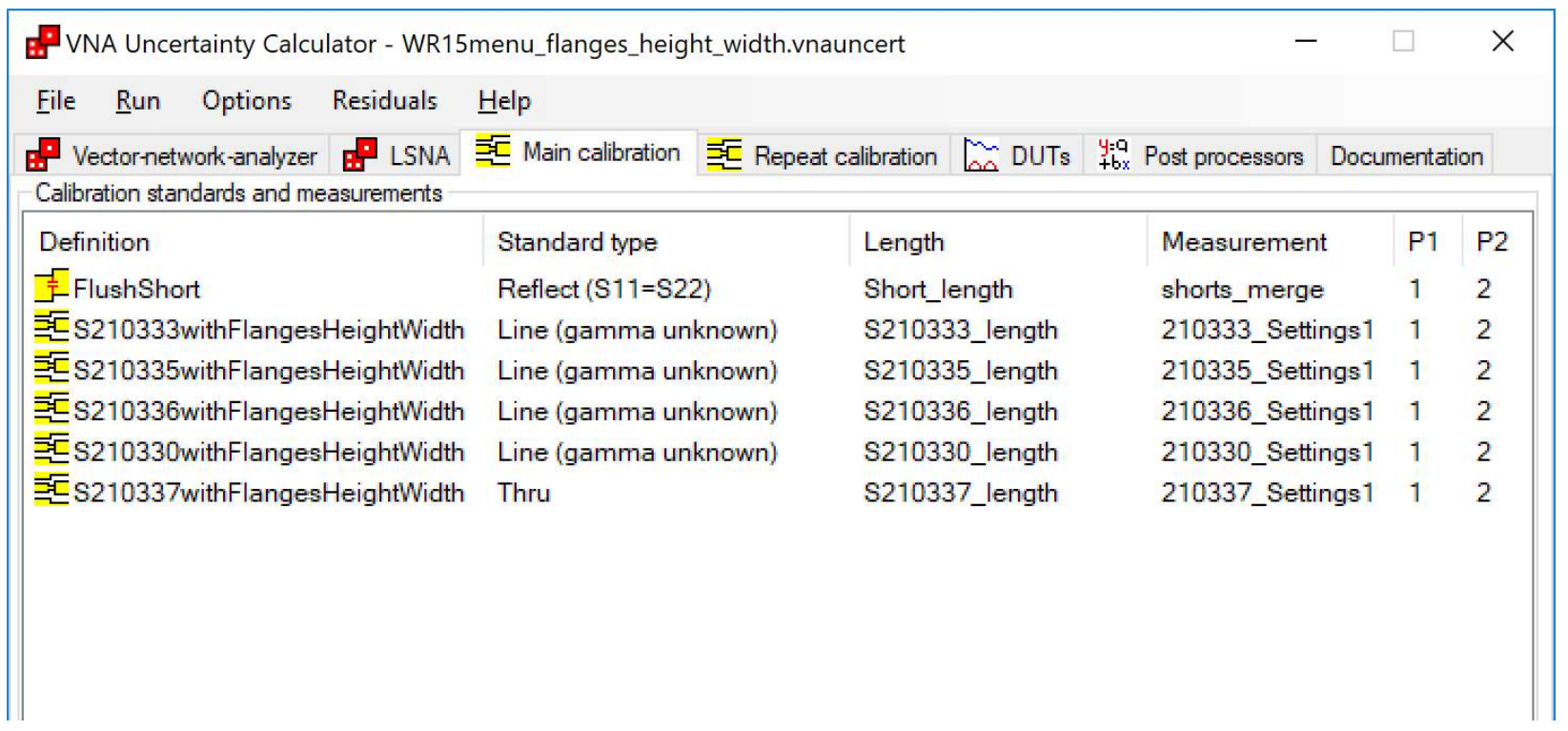

Fig. 12. MUF 'VNA Uncertainty Calculator' showing calibration standard definitions, standard types, lengths, and measurement files. 


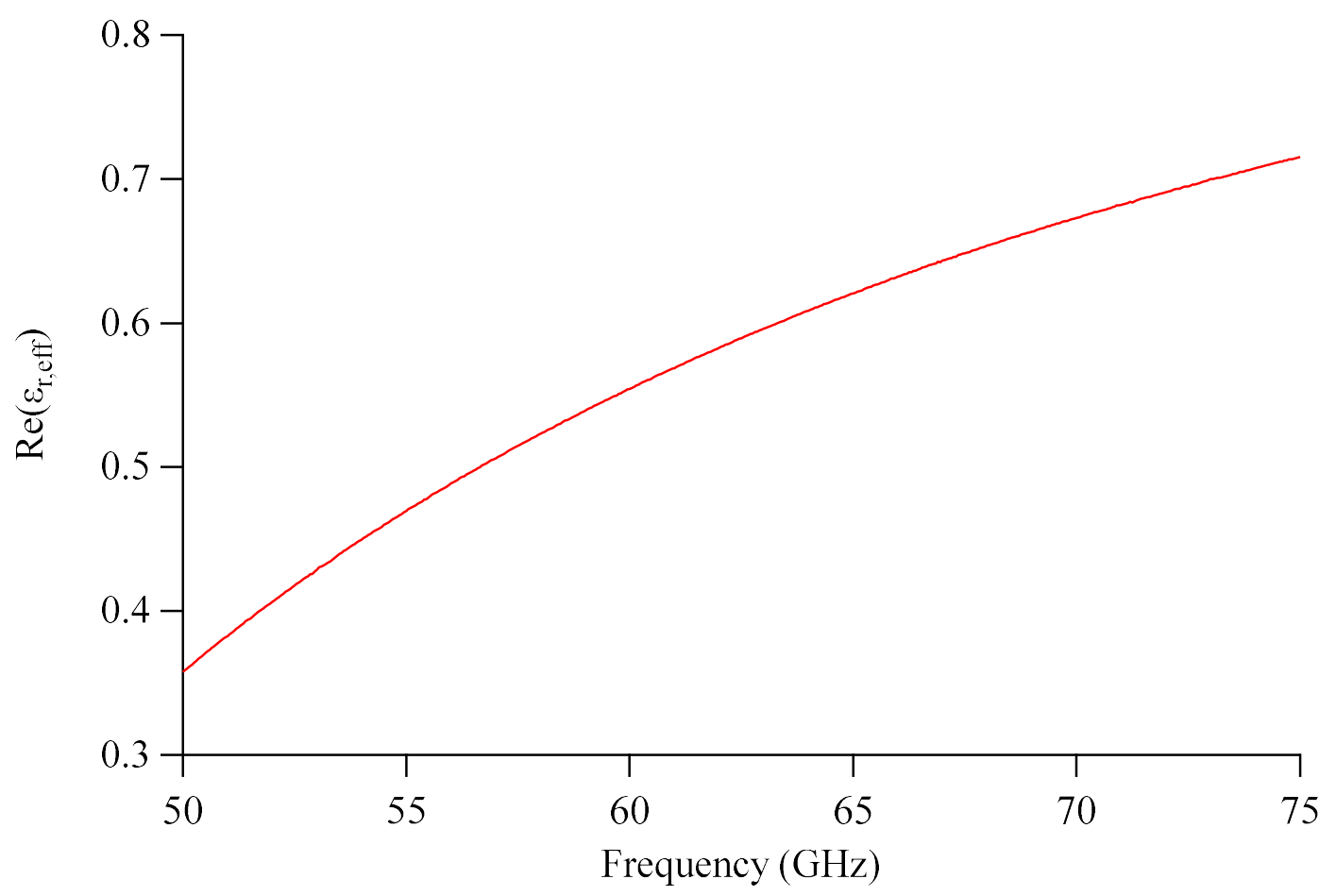

Fig. 13. Real part of $\varepsilon_{\text {r,eff }}$ obtained from the multiline TRL calibration.

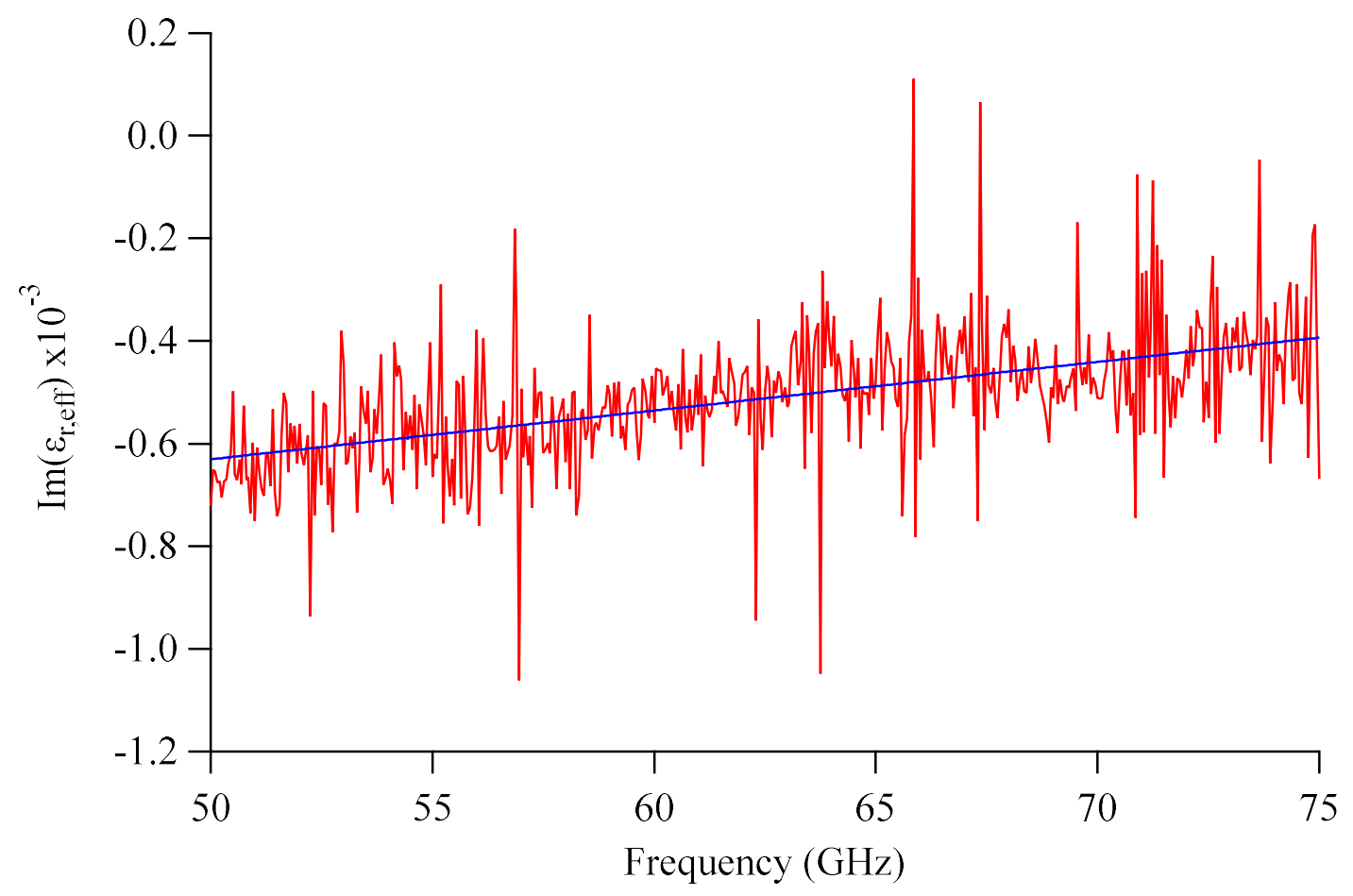

Fig. 14. Imaginary part of $\varepsilon_{\mathrm{r} \text {,eff }}$ obtained from the multiline TRL calibration. The measured values are shown in red and the straight-line fit is shown in blue. 


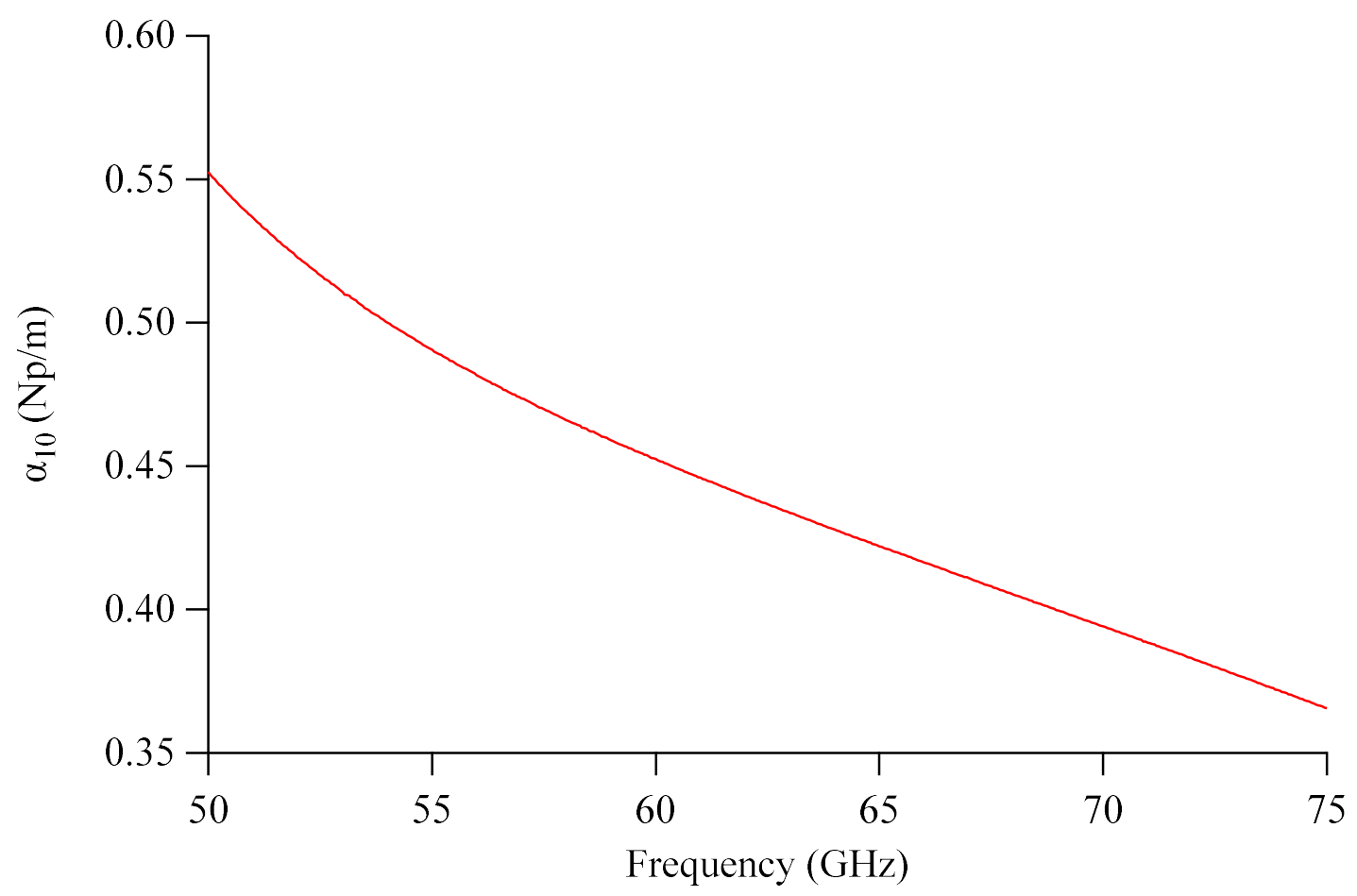

Fig. 15. Attenuation constant $\alpha_{10}$ calculated from $\varepsilon_{\text {r,eff }}$ using Eqs. (3.1), (3.2), and (3.3).

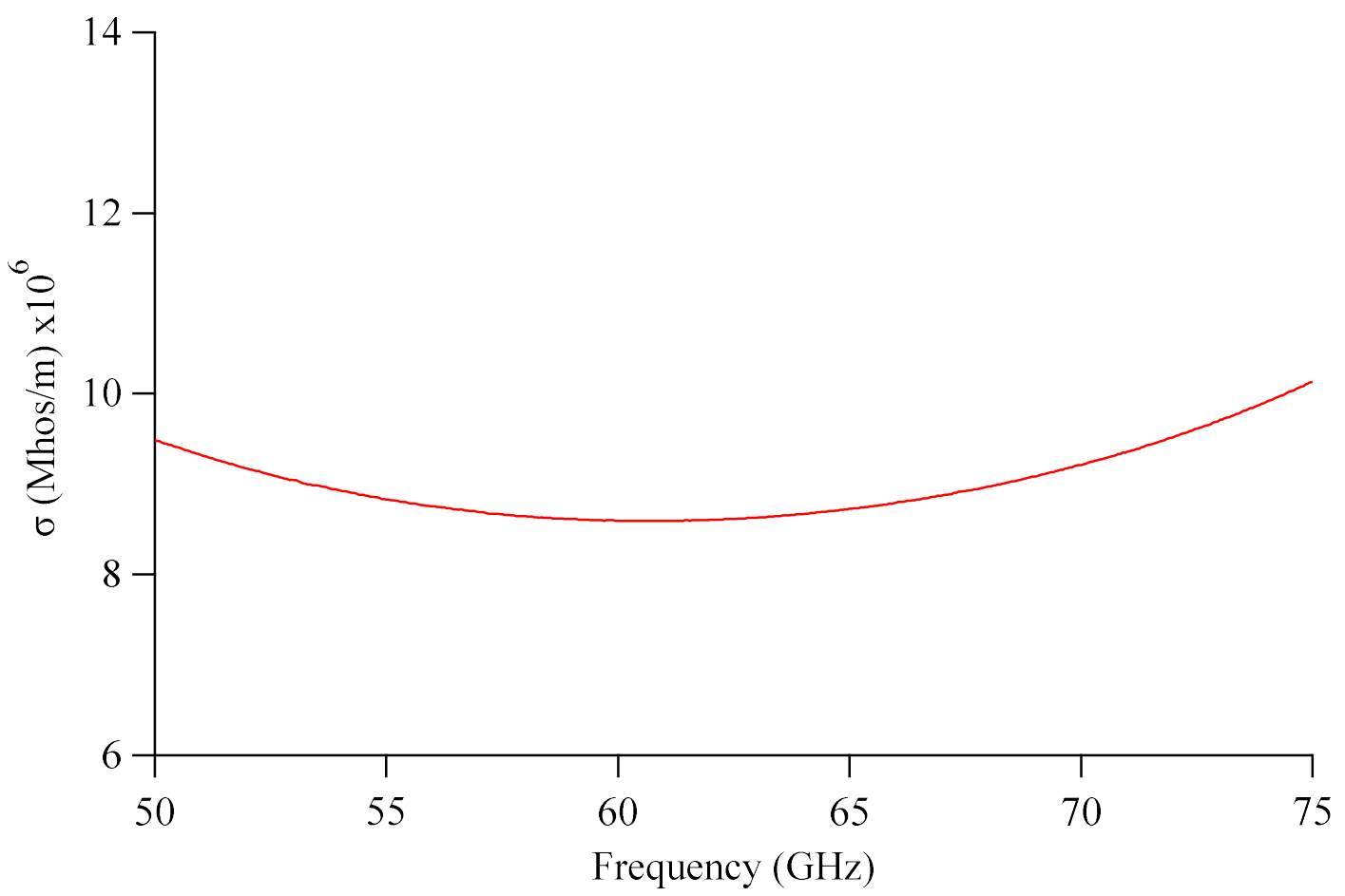

Fig. 16. Metal conductivity $\sigma$ calculated using Eqs. (3.4) and (3.5). 


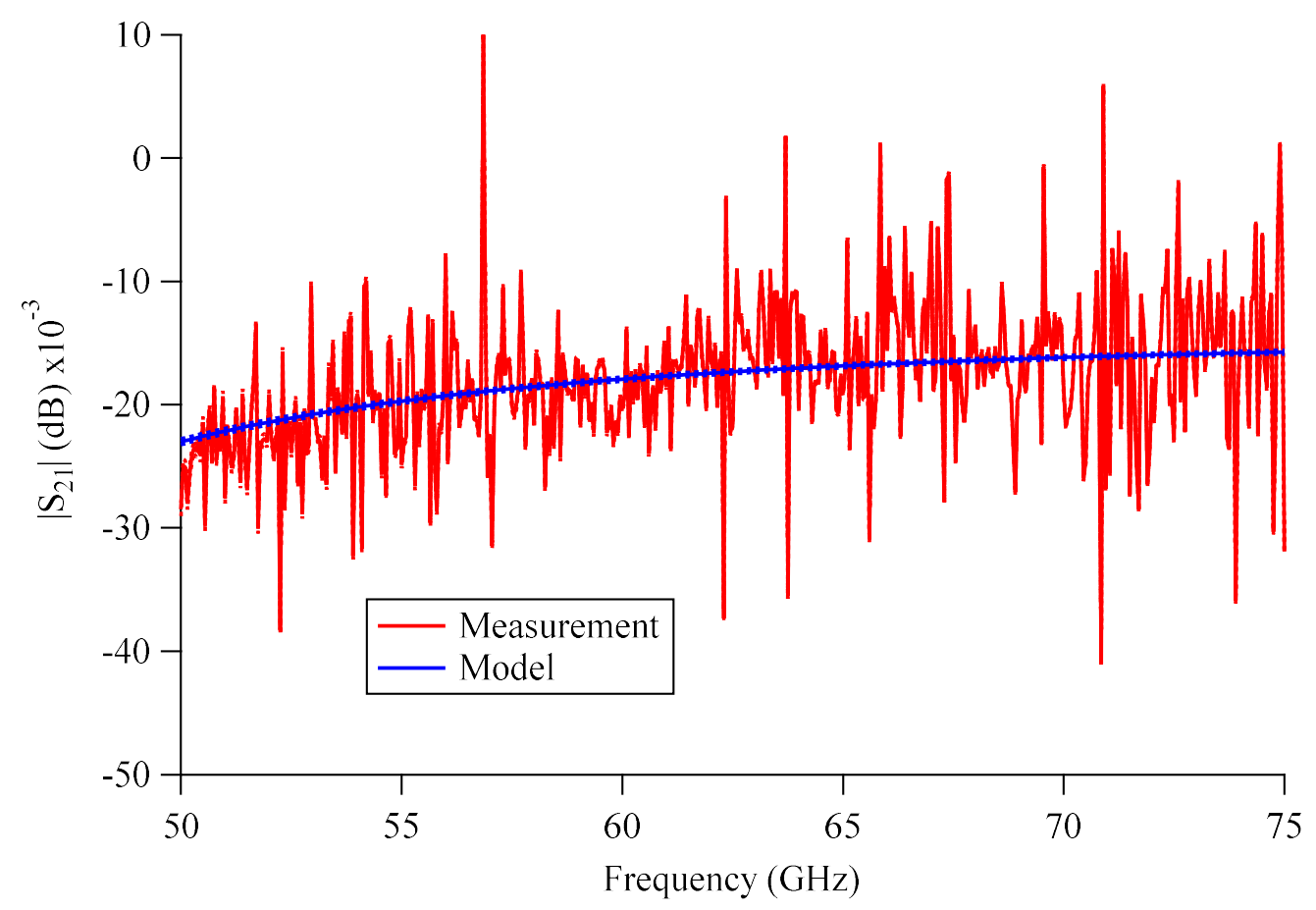

Fig. 17. Values of measured (red) and modeled (blue) $\left|S_{21}\right|$ and $95 \%$ confidence intervals for the calibrated $4.673 \mathrm{~mm}$ shim (S/N 210332). The dashed lines refer to confidence intervals.

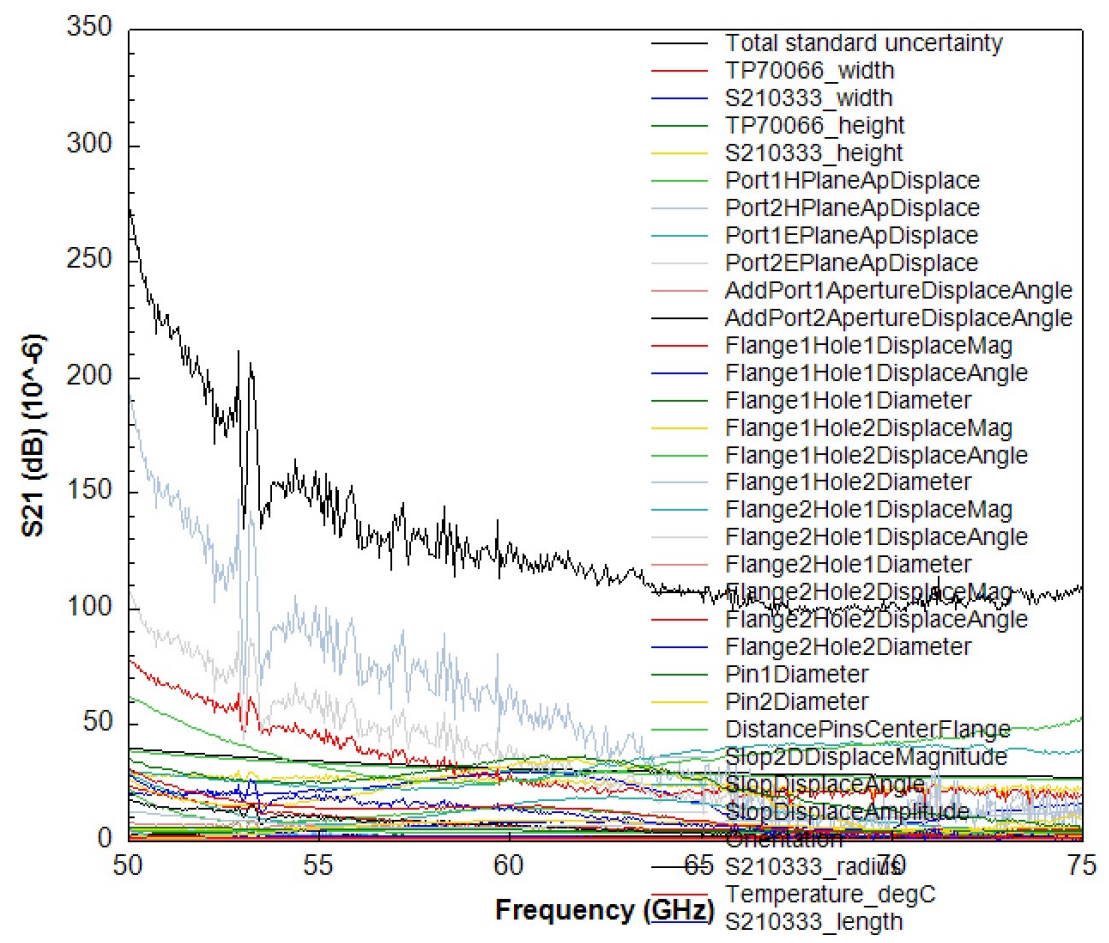

Fig. 18. Individual systematic uncertainty contributions and the total standard uncertainty for $\left|S_{21}\right|$ of the calibrated $4.673 \mathrm{~mm}$ shim (S/N 210332). 


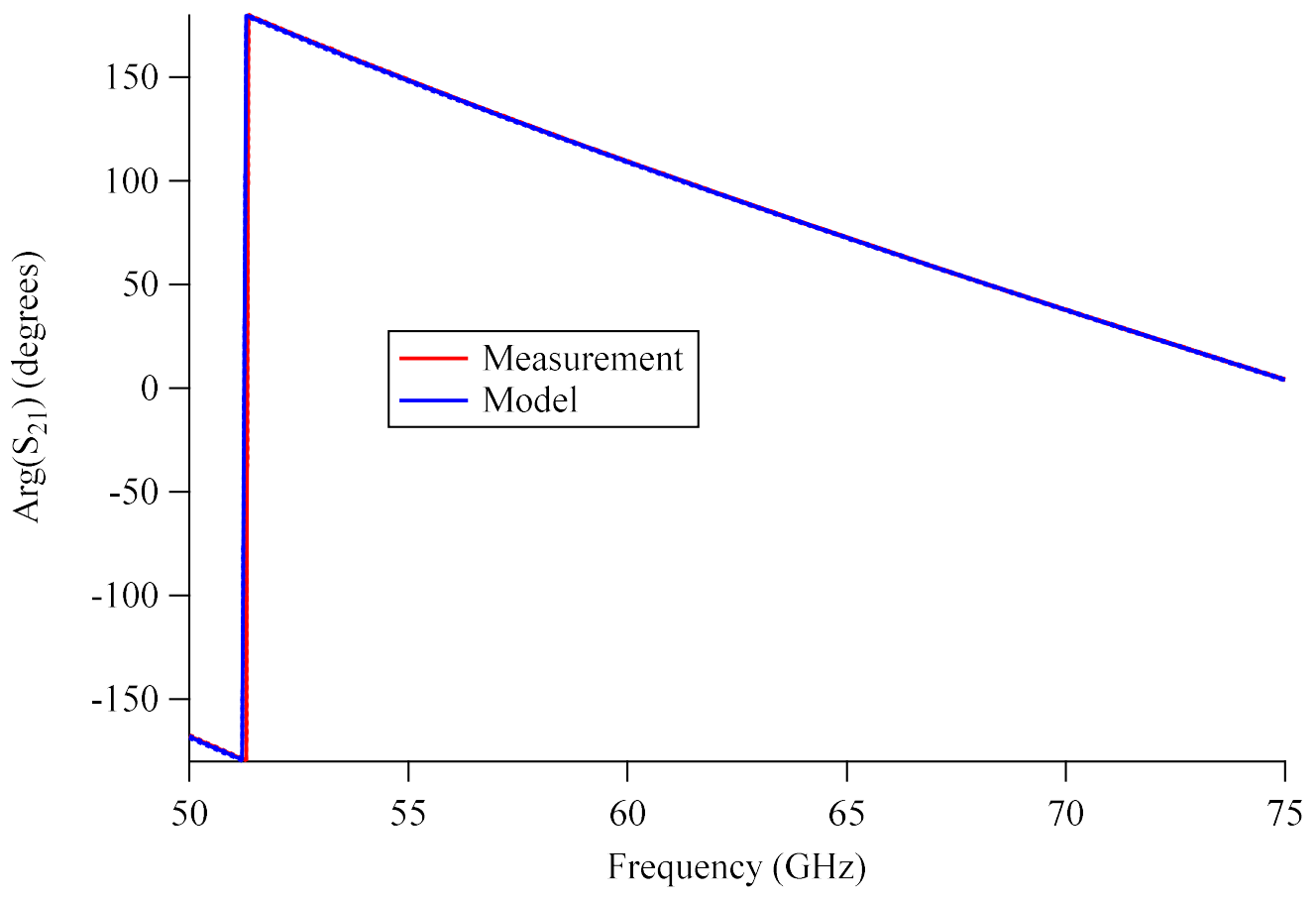

Fig. 19. Values of measured (red) and modeled (blue) $\operatorname{Arg}\left(S_{21}\right)$ and $95 \%$ confidence intervals for the calibrated $4.673 \mathrm{~mm}$ shim ( $\mathrm{S} / \mathrm{N} 210332)$. The dashed lines refer to confidence intervals.

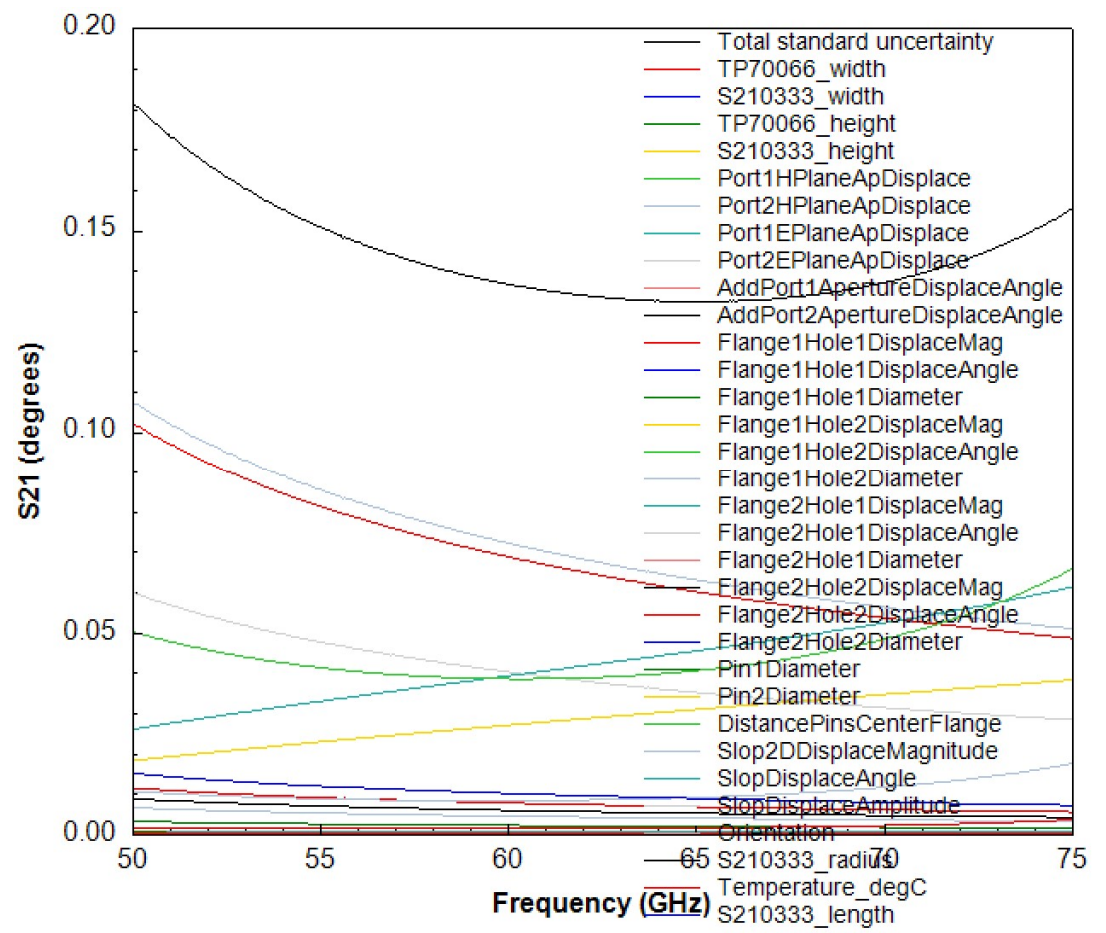

Fig. 20. Individual systematic uncertainty contributions and the total standard uncertainty for $\operatorname{Arg}\left(S_{21}\right)$ of the calibrated $4.673 \mathrm{~mm}$ shim $(\mathrm{S} / \mathrm{N} 210332)$. 


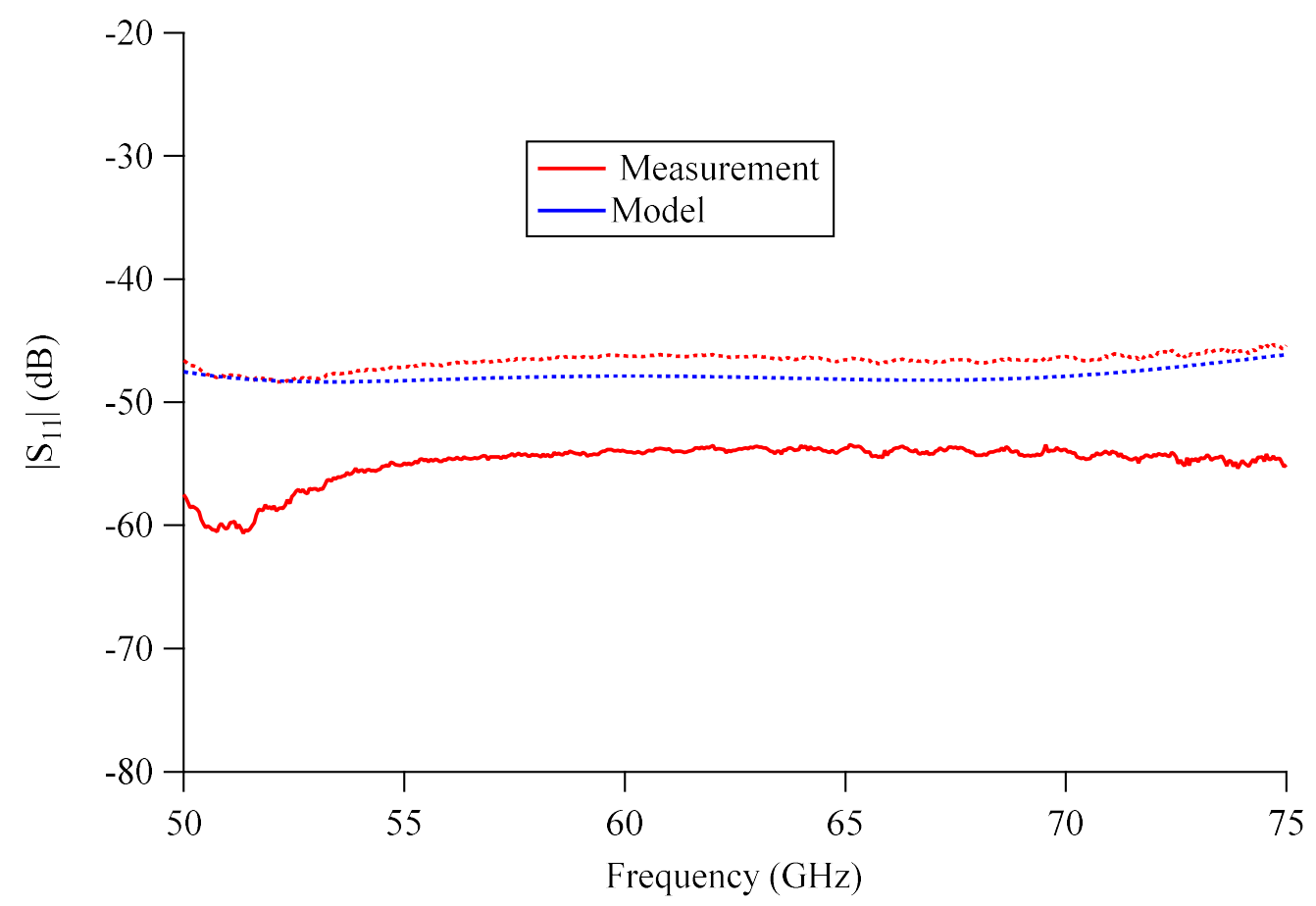

Fig. 21. Values of measured (red) and modeled (blue) $\left|S_{11}\right|$ and $95 \%$ confidence intervals for the calibrated $4.673 \mathrm{~mm}$ shim (S/N 210332). The dashed lines refer to confidence intervals.

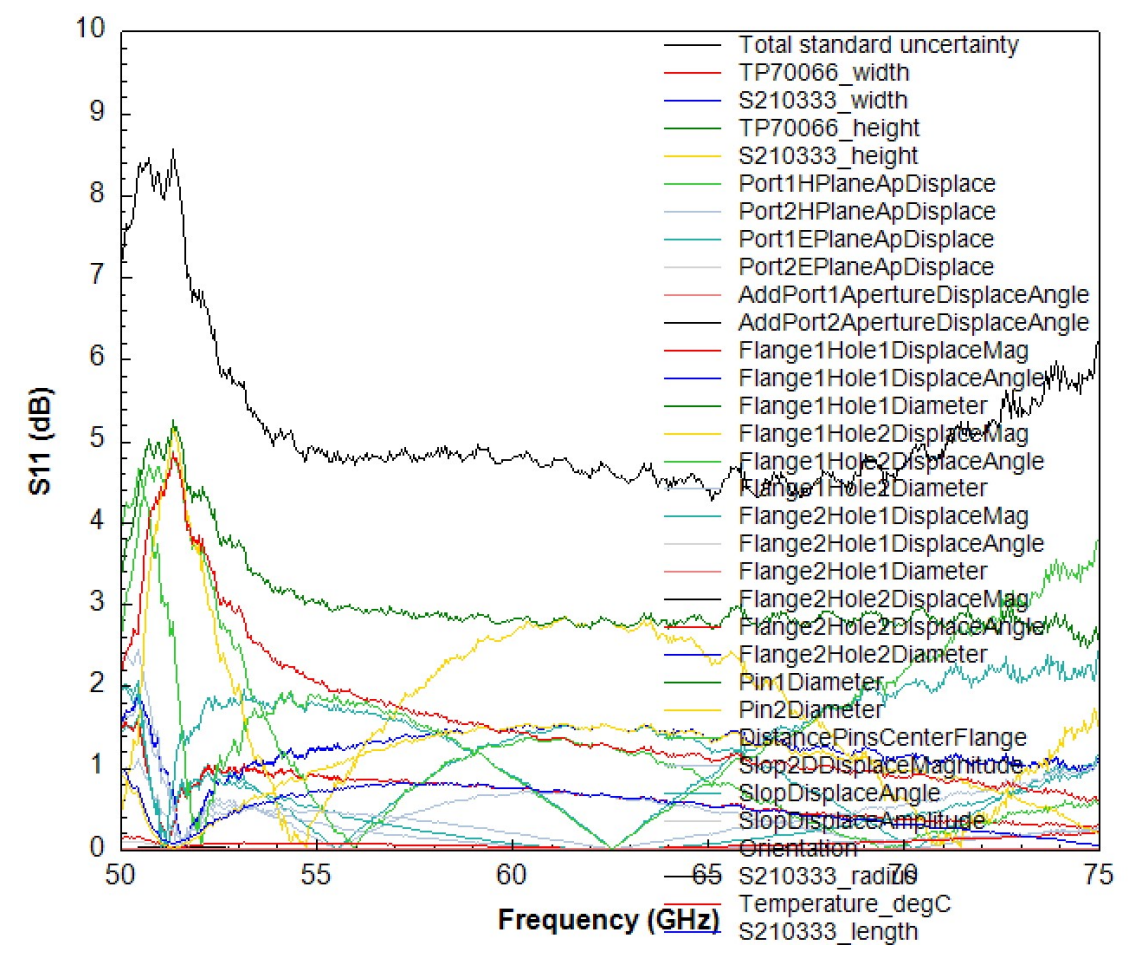

Fig. 22. Individual systematic uncertainty contributions and the total standard uncertainty for $\left|S_{11}\right|$ of the calibrated $4.673 \mathrm{~mm}$ shim (S/N 210332). 


\section{Acknowledgments}

The authors thank Jim Booth, Ari Feldman, Chris Holloway, and Mitch Wallis for their helpful comments.

\section{References}

[1] NIST Mission, Vision, Core Competencies, and Core Values. Available at https://www.nist.gov/about-nist/our-organization/mission-vision-values.

[2] Bureau International des Poids et Mesures (BIPM) (2012) International Vocabulary of Metrology - Basic and General Concepts and Associated Terms (VIM), Third Edition.

[3] Special Microwave and RF Scattering-Parameter Measurement Services SKU: 61290S. Available at: https://shop.nist.gov.

[4] Marks RB (1991) A multiline method of network analyzer calibration. IEEE Transactions on Microwave Theory and Techniques, 39 (7): 1205-1215.

[5] Marks RB (1997) Formulations of the basic vector network analyzer error model including switch terms. 50th ARFTG Microwave Measurement Conference, pp. 115-126.

[6] Jargon JA, Williams DF, Sanders A (2018) The relationship between switch-term-corrected scattering-parameters and wave-parameters measured with a two-port vector network analyzer. IEEE Microwave and Wireless Components Letters, 28 (10): 951-953.

[7] Marks RB, Williams DF (1991) Characteristic impedance determination using propagation constant measurement. IEEE Microwave and Guided Wave Letters, 1 (6): 141-143.

[8] Williams DF (2020) NIST Microwave Uncertainty Framework, Available at: https://www.nist.gov/services-resources/software/wafer-calibration-software.

[9] Collin RE (2001) Foundations for Microwave Engineering, Second Edition (Wiley-IEEE Press).

[10] Vacuum Magnetic Permeability, The NIST Reference on Constants, Units, and Uncertainty, Available at: https://physics.nist.gov/cgi-bin/cuu/Value?eqmu0.

[11] Vacuum Magnetic Permeability, The NIST Reference on Constants, Units, and Uncertainty, Available at: https://physics.nist.gov/cgi$\underline{\mathrm{bin} / \mathrm{cuu} / \text { Value? } \mathrm{ep} 0 \mid \text { search for=permittivty. }}$

[12] Anson WJ, Beatty RW, Kerns DM, Grandy, Jr. WT (1962) Investigations of the reflection from a junction of an ideal rectangular waveguide with one having rounded inside corners. PGMTT National Symposium Digest, pp. 27-31.

[13] Marks RB, Williams DF (1992) A general waveguide circuit theory. Journal of Research of the National Institute of Standards and Technology, 97 (5): 533-562. 
[14] Marcuvitz N (1951) Waveguide Handbook, First Edition (McGraw-Hill Book Company).

[15] Hunter JD (1984) The displaced rectangular waveguide junction and its uses as an adjustable reference reflection. IEEE Transactions on Microwave Theory and Techniques, 32 (4): 387-394.

[16] Williams, DF (2011) $500 \mathrm{GHz}-750 \mathrm{GHz}$ rectangular-waveguide vector-network-analyzer calibrations. IEEE Transactions on Terahertz Science and Technology, 1 (2): 364-377.

[17] Special Tests of Diameter SKU: 11050S. Available at: https://shop.nist.gov.

[18] Koepke A, Lafarge T, Possolo A, Toman B (2017) NIST consensus builder. Available at https://consensus.nist.gov.

[19] Koepke A, Lafarge T, Possolo A, Toman B (2017) Consensus building for interlaboratory studies, key comparisons, and meta-analysis. Metrologia, 54: S34-S62.

[20] Jargon JA, Arz U, Williams DF (2012) Characterizing WR-8 waveguide-to-CPW probes using two methods implemented within the NIST Uncertainty Framework. 80th ARFTG Microwave Measurement Conference.

[21] Jargon JA, Cho CH, Williams DF, Hale PD (2015) Physical models for $2.4 \mathrm{~mm}$ and 3.5 $\mathrm{mm}$ coaxial VNA calibration kits developed within the NIST Microwave Uncertainty Framework. 85th ARFTG Microwave Measurement Conference.

[22] Lewandowski A, Williams DF, Hale PD, Wang CM, Dienstfrey A (2010) Covariancematrix-based vector-network-analyzer uncertainty analysis for time-and frequency-domain measurements. IEEE Trans. Microwave Theory Tech., 58 (7): 1877-1886.

[23] Coefficients of Linear Thermal Expansion, Available at: www.engineeringtoolbox.com.

[24] The Institute of Electrical and Electronics Engineers (IEEE) Standard 1785.3 (2016) Recommended Practice for Rectangular Metallic Waveguides and Their Interfaces for Frequencies of $110 \mathrm{GHz}$ and Above - Part 3: Recommendations for Performance and Uncertainty Specifications.

[25] Williams DF (2016) P1785 RWG Uncertainty Calculator, Version 1.4.31.19543, Available at: https://www.nist.gov/services-resources/software/wafer-calibration-software. 


\section{Appendix: Pseudo-Code for Calculating Lateral and Angular Displacements}

We include the input quantities and pseudo-code used for calculating the lateral and angular displacements as discussed in IEEE Standard 1785.3-2016 [22] and implemented in the MUF [8] and the P1785 RWG Uncertainty Calculator [23].

$\%$ a_ideal $=$ Waveguide width a

$\%$ b_ideal $=$ Waveguide width $b$

\% a_offset_P1 = Port $1 \mathrm{H}$-Plane aperture (width) displacement

$\%$ a_offset_P2 = Port $2 \mathrm{H}$-Plane aperture (width) displacement

$\%$ b_offset_P1 = Port $1 \mathrm{E}$-Plane aperture (height) displacement

$\%$ b_offset_P2 = Port 2 E-Plane aperture (height) displacement

$\%$ offsetmag_A1 = Additional Port 1 aperture 2-D displacement magnitude

$\%$ phase_A1 = Additional Port 1 aperture displacement angle [0, 2*PI]

$\%$ offsetmag_A2 = Additional Port 2 aperture 2-D displacement magnitude

$\%$ phase_A2 = Additional Port 2 aperture displacement angle [0, 2*PI]

$\%$ offsetmag_F1H1 = Flange 1 hole 12 -D displacement magnitude

$\%$ phase_F1H1 $=$ Flange 1 hole 1 displacement angle $[0,2 * \mathrm{PI}]$

$\%$ diameter_F1H1 = Flange 1 hole 1 diameter

$\%$ offsetmag_F1H2 = Flange 1 hole 2 2-D displacement magnitude

$\%$ phase_F1H2 $=$ Flange 1 hole 2 displacement angle [0, 2*PI]

$\%$ diameter_F1H2 = Flange 1 hole 2 diameter

$\%$ offsetmag_F2H1 = Flange 2 hole $12-\mathrm{D}$ displacement magnitude

$\%$ phase_F2H1 = Flange 2 hole 1 displacement angle [0, 2*PI]

$\%$ diameter_F2H1 = Flange 2 hole 1 diameter

$\%$ offsetmag_F2H2 = Flange 2 hole 2 2-D displacement magnitude

$\%$ phase_F2H2 = Flange 2 hole 2 displacement angle [0, $\left.{ }^{*} \mathrm{PI}\right]$

$\%$ diameter_F2H2 = Flange 2 hole 2 diameter

$\%$ Pin1Diam $=$ Pin 1 diameter

$\%$ Pin2Diam $=$ Pin 2 diameter

$\%$ PinDistanceFromCenter $=$ Distance of pins from center of flange

$\%$ offsetmag_Slop $=$ Slop 2-D displacement magnitude $[-1,1]$

$\%$ phase_Slop $=$ Slop displacement angle [0, 2*PI]

$\%$ offsetang_Slop $=$ Slop displacement amplitude $[-1,1]$

$\%$ a_orient $=$ Orientation [+/- 1 flips orientation 180 degrees] 
$\%$ Create a scattering-parameter instance.

$\%$ The 9 columns are:

\% Frequency, $\operatorname{Re}(\mathrm{S} 11), \operatorname{Im}(\mathrm{S} 11), \operatorname{Re}(\mathrm{S} 21), \operatorname{Im}(\mathrm{S} 21), \operatorname{Re}(\mathrm{S} 12), \operatorname{Im}(\mathrm{S} 12), \operatorname{Re}(\mathrm{S} 22), \operatorname{Im}(\mathrm{S} 22)$

mySParams = RealMatrix(FrequencyList, 9, ".s2p")

$\%$ Initialize a $2 \times 2$ array of complex numbers.

S_B $=$ CmplxMatrix $(2,2)$

\% Transform tolerance zones into $\mathrm{x}, \mathrm{y}$ coordinates.

$A 1 x=$ a_offset_P1 + offsetmag_A1 $*$ cos(phase_A1)

$A 1 y=b \_o f f s e t \_P 1+$ offsetmag_A1 $* \sin ($ phase_A1)

$A 2 x=$ a_offset_P2 + offsetmag_A2 $* \cos ($ phase_A2)

$A 2 y=b \_o f f s e t \_P 2+$ offsetmag_A2 $* \sin ($ phase_A2)

$\mathrm{F} 1 \mathrm{H} 1 \mathrm{x}=$ offsetmag_F1H1 $* \cos ($ phase_F1H1)

$\mathrm{F} 1 \mathrm{H} 1 \mathrm{y}=$ offsetmag_F1H1 $* \sin ($ phase_F1H1)

$\mathrm{F} 1 \mathrm{H} 2 \mathrm{x}=$ offsetmag_F1H2 $* \cos ($ phase_F1H2)

$\mathrm{F} 1 \mathrm{H} 2 \mathrm{y}=$ offsetmag_F1H2 $* \sin ($ phase_F1H2)

$\mathrm{F} 2 \mathrm{H} 1 \mathrm{x}=$ offsetmag_F2H1 $* \cos$ (phase_F2H1)

$\mathrm{F} 2 \mathrm{H} 1 \mathrm{y}=$ offsetmag_F2H1 $* \sin ($ phase_F2H1)

$\mathrm{F} 2 \mathrm{H} 2 \mathrm{x}=$ offsetmag_F2H $2 * \cos$ (phase_F2H2)

$\mathrm{F} 2 \mathrm{H} 2 \mathrm{y}=$ offsetmag_F $2 \mathrm{H} 2 * \sin ($ phase_F2H2)

phase_Slopx $=$ offsetmag_Slop $*$ cos(phase_Slop)

phase_Slopy $=$ offsetmag_Slop $* \sin ($ phase_Slop)

\% Add in the PinDistanceFromCenter to the hole positions.

$\mathrm{F} 1 \mathrm{H} 1 \mathrm{y}=\mathrm{F} 1 \mathrm{H} 1 \mathrm{y}+$ PinDistanceFromCenter

$\mathrm{F} 2 \mathrm{H} 1 \mathrm{y}=\mathrm{F} 2 \mathrm{H} 1 \mathrm{y}+$ PinDistanceFromCenter

$\mathrm{F} 1 \mathrm{H} 2 \mathrm{y}=\mathrm{F} 1 \mathrm{H} 2 \mathrm{y}-$ PinDistanceFromCenter

$\mathrm{F} 2 \mathrm{H} 2 \mathrm{y}=\mathrm{F} 2 \mathrm{H} 2 \mathrm{y}-$ PinDistanceFromCenter 
$\%$ Find the aperture offset.

A2AperturePosition $=$ TwoPinTransform(F1H1x, F1H1y, diameter_F1H1 / 2.0, F1H2x, F1H2y, diameter_F1H2 / 2.0, F2H1x, F2H1y, diameter_F2H1 / 2.0, F2H2x, F2H2y, diameter_F2H2 / 2.0, Pin1Diam / 2.0, Pin2Diam / 2.0, phase_Slopx, phase_Slopy, offsetang_Slop, a_orient > $0.0, \mathrm{~A} 2 \mathrm{x}, \mathrm{A} 2 \mathrm{y})$

$\%$ Find the aperture angle.

A2ApertureAngle $=$ TwoPinTransform $(F 1 H 1 x, F 1 H 1 y$, diameter_F1H1 / 2.0, F1H2x, F1H2y, diameter_F1H2 / 2.0, F2H1x, F2H1y, diameter_F2H1 / 2.0, F2H2x, F2H2y, diameter_F2H2 / 2.0, Pin1Diam / 2.0, Pin2Diam / 2.0, phase_Slopx, phase_Slopy, offsetang_Slop, a_orient > $0.0, A 2 x, 1.0+A 2 y)$

\% Small angle approximation.

A2Angle $=(180.0 / \mathrm{PI}) *($ A2ApertureAngle(0) - A2AperturePosition(0)) $/$

(A2ApertureAngle(1) - A2AperturePosition(1))

$\%$ Flip the angle if the aperture got flipped upside down.

If A2ApertureAngle(1) - A2AperturePosition $(1)<0.0$ Then A2Angle $=-$ A2Angle

$\%$ Correlated pin offsets.

a_offset $=$ A2xNew $-A 1 x$

b_offset $=$ A2yNew - A1y

If Abs(b_offset) $>0.25 *$ Abs(b_ideal) Then

Report "E-plane offset is too large compared to waveguide height to give accurate results."

If Abs(a_offset) $>0.25 *$ Abs(a_ideal) Then

Report "H-plane offset is too large compared to waveguide height to give accurate results."

If a_ideal $>1.02 *$ Lambda0 Then

Report "Frequency is too low to calculate H-plane offset accurately."

If a_ideal $<0.55 *$ Lambda0 Then

Report "Frequency is too high to calculate H-plane offset accurately."

If $A b s$ (A2Angle) $>6.0$ Then

Report "Angle is too high to calculate angle offset accurately." 
$\%$ Step through the frequencies and generate the scattering parameters of the junction.

For $\mathrm{k}=1$ To NumberOfFrequencies

$\%$ Set up the frequencies and other parameters.

$\mathrm{FGHz}=\operatorname{mySParams}(\mathrm{k}, 1)$

$\%$ Call the routines.

Call WidthOffset_Hunter(B_YO_W, a_offset, a_ideal, b_ideal, FGHz)

Call HeightOffset_Hunter(B_YO_H, b_offset, b_ideal, a_ideal, FGHz)

Call AngleOffset(B_Y0_A, A2Angle, a_ideal, FGHz)

B_YO_effective $=$ B_YO_W + B_YO_H + B_YO_A

\% Calculate the impedance transform and admittance.

S_B = SParams_B_YO(B_YO_effective)

$\operatorname{mySParams}(k)=$ S_B

Next $k$

Cascade the input scattering parameters if they are supplied.

mySParams = mySParams.CascadeSParameters(SParamsIn) 
Subroutine WidthOffset_Hunter(B_YO_effective, a_Offset, a_Ideal, b_Ideal, FGHz)

$$
\begin{aligned}
& x i=\text { a_Ideal / Lambda0 } \\
& \text { alpha }=0.7 \\
& \text { tau = Abs(a_Offset / a_Ideal) } \\
& \mathrm{u}(0)=1.75 \\
& u(1)=-0.332 \\
& u(2)=-2.71 \\
& u(3)=-3.57 \\
& v(0)=0.635 \\
& v(1)=-1.562 \\
& v(2)=0.44 \\
& v(3)=-7.63 \\
& \text { B_YO_effective }=0.0 \\
& \mathrm{~s} 1=0.0 \\
& \mathrm{~s} 2=0.0 \\
& \text { For } \mathrm{k} 1=0 \text { To } 3 \\
& \mathrm{~s} 1=\mathrm{s} 1+\mathrm{u}(\mathrm{k} 1)^{*}\left((\mathrm{xi}-\mathrm{alpha})^{\wedge} \mathrm{k} 1\right) \\
& s 2=s 2+v(k 1)^{*}\left((x i-\text { alpha })^{\wedge} k 1\right)
\end{aligned}
$$

End Subroutine 
Subroutine HeightOffset_Hunter(B_YO_effective, b_Offset, b_Ideal, a_Ideal, FGHz)

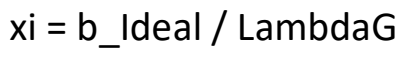

$$
\begin{aligned}
& \text { alpha }=0.3 \\
& \text { tau = Abs(b_Offset / b_Ideal) } \\
& \mathrm{u}(0)=1.833 \\
& u(1)=0.276 \\
& u(2)=0.73 \\
& u(3)=0.0 \\
& v(0)=0.293 \\
& v(1)=2.133 \\
& v(2)=0.78 \\
& v(3)=19.69 \\
& \text { B_YO_effective }=0.0 \\
& \mathrm{~s} 1=0.0 \\
& \mathrm{~s} 2=0.0 \\
& \text { For } \mathrm{k} 1=0 \text { To } 3 \\
& \mathrm{~s} 1=\mathrm{s} 1+\mathrm{u}(\mathrm{k} 1)^{*}\left((\mathrm{xi}-\mathrm{alpha})^{\wedge} \mathrm{k} 1\right) \\
& s 2=s 2+v(k 1) *\left((x i-a l p h a)^{\wedge} k 1\right) \\
& \text { gamma }=10.0^{\wedge}(\mathrm{s} 1 * \log 10(\text { tau })+\mathrm{s} 2) \\
& \text { B_YO_effective }=2.0 * \text { gamma } / \text { Math.Sqrt(1.0 }- \text { gamma } * \text { gamma })
\end{aligned}
$$

End Subroutine

Subroutine AngleOffset(B_Y0_effective, Angle_Offset, a_Ideal, FGHz)

B_YO_effective $=-\left(0.000225 *\left(\right.\right.$ Angle_Offset $\left.{ }^{\wedge} 2\right)+\left(0.0+0.0049 *\left(\right.\right.$ Angle_Offset ${ }^{\wedge}$ $2))^{*}\left(\right.$ Abs $\left.\left.(\text { eps }-0.9)^{\wedge} 2\right)\right)$

End Subroutine 
Function TwoPinTransform(F1H1x, F1H1y, F1H1r, F1H2x, F1H2y, F1H2r, F2H1x, F2H1y, $\mathrm{F} 2 \mathrm{H} 1 \mathrm{r}, \mathrm{F} 2 \mathrm{H} 2 \mathrm{x}, \mathrm{F} 2 \mathrm{H} 2 \mathrm{y}, \mathrm{F} 2 \mathrm{H} 2 \mathrm{r}, \mathrm{P} 1 \mathrm{r}, \mathrm{P} 2 \mathrm{r}, \mathrm{Z1}$, Z2, Z3, Flipped, Aperturelnputx, Aperturelnputy)

$\% \mathrm{~F} 1 \mathrm{H} 1 \mathrm{x}=\mathrm{x}$ coordinate of hole 1 in flange 1.

$\% \mathrm{~F} 1 \mathrm{H} 1 \mathrm{y}=\mathrm{y}$ coordinate of hole 1 in flange 1.

$\% \mathrm{~F} 1 \mathrm{H} 1 \mathrm{r}=$ radius of hole 1 in flange 1.

$\% \mathrm{~F} 1 \mathrm{H} 2 \mathrm{x}=\mathrm{x}$ coordinate of hole 2 in flange 1.

$\% \mathrm{~F} 1 \mathrm{H} 2 \mathrm{y}=\mathrm{y}$ coordinate of hole 2 in flange 1.

$\% \mathrm{~F} 1 \mathrm{H} 2 \mathrm{r}=$ radius of hole 2 in flange 1.

$\% \mathrm{~F} 2 \mathrm{H} 1 \mathrm{x}=\mathrm{x}$ coordinate of hole 1 in flange 2 .

$\% \mathrm{~F} 2 \mathrm{H} 1 \mathrm{y}=\mathrm{y}$ coordinate of hole 1 in flange 2 .

$\% \mathrm{~F} 2 \mathrm{H} 1 \mathrm{r}=$ radius of hole 1 in flange 2 .

$\% \mathrm{~F} 2 \mathrm{H} 2 \mathrm{x}=\mathrm{x}$ coordinate of hole 2 in flange 2.

$\% \mathrm{~F} 2 \mathrm{H} 2 \mathrm{y}=\mathrm{y}$ coordinate of hole 2 in flange 2 .

$\% \mathrm{~F} 2 \mathrm{H} 2 \mathrm{r}=$ radius of hole 2 in flange 2 .

$\% \mathrm{P} 1 \mathrm{r}=$ radius of pin in hole 1 .

$\% \mathrm{P} 2 \mathrm{r}=$ radius of pin in hole 2 .

$\% \mathrm{Z1}=\mathrm{x}$ coordinate of vector lying in unit circle that determines position in smaller hole.

$\% \mathrm{ZZ}=\mathrm{y}$ coordinate of vector lying in unit circle that determines position in smaller hole.

$\% Z 3$ = value between $+/-1$ that determines position in larger hole.

$\%$ Flipped $=$ When set true, this flips the second flange by 180 degrees.

$\%$ Aperturelnputx $=x$ coordiante of the input position on the second flange.

$\%$ Aperturelnputy $=y$ coordiante of the input position on the second flange.

$\%$ Returns $x$ and $y$ coordinates of the output position on the first flange.

$\%$ There is no checking to make sure that the holes are big enough, etc.

$\%$ This approach does not take into account wedging of the pin between holes if the distance between holes in different on the two flanges.

$\%$ This results in a sort of worst-case angle variation.

$\%$ Aperturelnputx and Aperturelnputy are the $x$ and $y$ of the (aperture) position in the coordinate system of the second flange.

$\%$ ApertureOutput will have the $\mathrm{x}$ and $\mathrm{y}$ of the (aperture) position in the coordinate system of the first flange.

$\% \mathrm{vt}$ is the temporary location.

\% Figure out which flange has the least space to move (smallest slop).

$\mathrm{H} 1 \mathrm{Slop}=\mathrm{F} 1 \mathrm{H} 1 \mathrm{r}+\mathrm{F} 2 \mathrm{H} 1 \mathrm{r}-2.0 * \mathrm{P} 1 \mathrm{r}$

$\mathrm{H} 2 \mathrm{Slop}=\mathrm{F} 1 \mathrm{H} 2 \mathrm{r}+\mathrm{F} 2 \mathrm{H} 2 \mathrm{r}-2.0 * \mathrm{P} 2 \mathrm{r}$ 
\% If we flip flange 2 , then hole 1 on flange 1 now mates with hole 2 on flange 2.

If Flipped Then

$$
\begin{aligned}
& \mathrm{H} 1 \mathrm{Slop}=\mathrm{F} 1 \mathrm{H} 1 \mathrm{r}+\mathrm{F} 2 \mathrm{H} 2 \mathrm{r}-2.0 * \mathrm{P} 1 \mathrm{r} \\
& \mathrm{H} 2 \mathrm{Slop}=\mathrm{F} 1 \mathrm{H} 2 \mathrm{r}+\mathrm{F} 2 \mathrm{H} 1 \mathrm{r}-2.0 * \mathrm{P} 2 \mathrm{r}
\end{aligned}
$$

End If

If H1Slop $<0.0$ Then H1Slop $=0.0$

If $\mathrm{H} 2 \mathrm{Slop}<0.0$ Then $\mathrm{H} 2 \mathrm{Slop}=0.0$

$\%$ Get all the coordinates into complex numbers.

$\mathrm{u} 1=\mathrm{Cmplx}(\mathrm{F} 1 \mathrm{H} 1 \mathrm{x}, \mathrm{F} 1 \mathrm{H} 1 \mathrm{y})$

$\mathrm{u} 2=\mathrm{Cmplx}(\mathrm{F} 1 \mathrm{H} 2 \mathrm{x}, \mathrm{F} 1 \mathrm{H} 2 \mathrm{y})$

$\mathrm{v} 1=\mathrm{Cmplx}(-\mathrm{F} 2 \mathrm{H} 1 \mathrm{x}, \mathrm{F} 2 \mathrm{H} 1 \mathrm{y})$

$\mathrm{v} 2=\mathrm{Cmp} \mid x(-\mathrm{F} 2 \mathrm{H} 2 \mathrm{x}, \mathrm{F} 2 \mathrm{H} 2 \mathrm{y})$

\% Flip flange two around to mate with flange 1.

v0 = Cmplx(-Aperturelnputx, Aperturelnputy)

\% Flip everything on flange two by 180 degrees, and now call hole 1 hole 2 and vice versa.

If Flipped Then

$$
\begin{aligned}
& \mathrm{vt}=\mathrm{v} 1 \\
& \mathrm{v} 1=-\mathrm{v} 2 \\
& \mathrm{v} 2=-\mathrm{vt} \\
& \mathrm{v} 0=-\mathrm{v} 0
\end{aligned}
$$

End If

$\%$ See how the input aperture position maps from the coordinate ssytem of the second flange to the coordinate system of the first flange.

If H1Slop <= H2Slop Then \% Reference hole is hole 1. Hole 2 sets the angle.

$$
\begin{aligned}
& \mathrm{ZZ}=\mathrm{H} 1 \mathrm{Slop} * \mathrm{Cmplx}(\mathrm{Z1}, \mathrm{Z2}) \quad \%(\mathrm{Z1}, \mathrm{Z2}) \text { in the unit circle, sets origin } \\
& \mathrm{Zmax}=\mathrm{H} 2 \mathrm{Slop} * \mathrm{Z3} \quad \%-1<Z 3<1 \text {, sets angle theta } \\
& \text { eitheta }=(\mathrm{u} 2+Z \max -(\mathrm{u} 1+\mathrm{ZZ})) /(\mathrm{v} 2-\mathrm{v} 1) \quad \% \text { Has magnitude and phase } \\
& \text { eitheta }=\text { eitheta } / \text { Abs(eitheta) } \quad \% \text { Keep the phase } \\
& \text { ApertureOut }=(v 0-v 1) * \text { eitheta }+(u 1+Z Z)
\end{aligned}
$$


Else

\% Reference hole is hole 2 . Hole 1 sets the angle.

$$
\begin{aligned}
& \mathrm{ZZ}=\mathrm{H} 2 \mathrm{Slop} * \mathrm{Cmplx}(\mathrm{Z1}, \mathrm{Z2}) \quad \%(\mathrm{Z1}, \mathrm{Z2}) \text { in unit circle, sets origin } \\
& \text { Zmax }=\text { H1Slop } * Z 3 \quad \%-1<Z 3<1 \text {, sets angle theta } \\
& \text { eitheta }=(\mathrm{u} 1+\mathrm{Zmax}-(\mathrm{u} 2+\mathrm{ZZ})) /(\mathrm{v} 1-\mathrm{v} 2) \quad \% \text { Has magnitude and phase } \\
& \text { eitheta }=\text { eitheta } / \text { Abs(eitheta) } \quad \% \text { Keep the phase } \\
& \text { ApertureOut }=(v 0-v 2) * \text { eitheta }+(u 2+Z Z)
\end{aligned}
$$

End If

ApertureOutput $(0)=$ ApertureOut. Re

ApertureOutput $(1)$ = ApertureOut.Im

Return ApertureOutput

End Function 